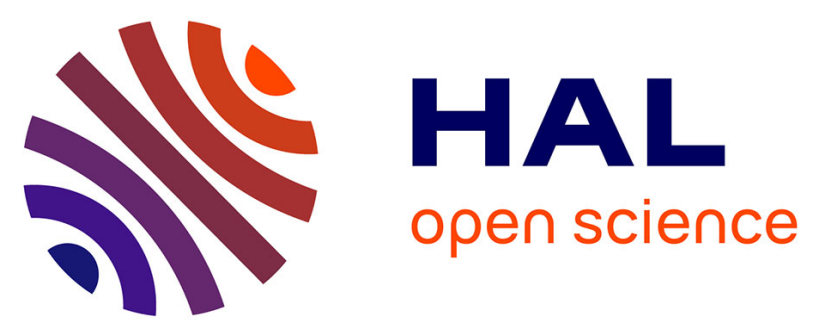

\title{
Longitudinal liver proteome profiling in dairy cows during the transition from gestation to lactation: Investigating metabolic adaptations and their interactions with fatty acids supplementation via repeated measurements ANOVA-simultaneous component analysis
}

Arash Veshkini, Harald M. Hammon, Helga Sauerwein, Arnulf Tröscher, Didier Viala, Mylène Delosière, Fabrizio Ceciliani, Sébastien Déjean, Muriel Bonnet

\section{To cite this version:}

Arash Veshkini, Harald M. Hammon, Helga Sauerwein, Arnulf Tröscher, Didier Viala, et al.. Longitudinal liver proteome profiling in dairy cows during the transition from gestation to lactation: Investigating metabolic adaptations and their interactions with fatty acids supplementation via repeated measurements ANOVA-simultaneous component analysis. Journal of Proteomics, 2022, 252, pp.104435. 10.1016/j.jprot.2021.104435 . hal-03473221

\section{HAL Id: hal-03473221 \\ https://hal.inrae.fr/hal-03473221}

Submitted on 10 Dec 2021

HAL is a multi-disciplinary open access archive for the deposit and dissemination of scientific research documents, whether they are published or not. The documents may come from teaching and research institutions in France or abroad, or from public or private research centers.
L'archive ouverte pluridisciplinaire HAL, est destinée au dépôt et à la diffusion de documents scientifiques de niveau recherche, publiés ou non, émanant des établissements d'enseignement et de recherche français ou étrangers, des laboratoires publics ou privés. 

lactation: Investigating metabolic adaptations and their interactions with fatty acids supplementation via repeated measurements ANOVA-simultaneous component analysis

5 Authors:

6 Arash Veshkini ${ }^{1,2,3,4}$, Harald M. Hammon ${ }^{2 *}$, Helga Sauerwein ${ }^{1}$, Arnulf Tröscher ${ }^{5}$, Didier Viala ${ }^{3}$, Mylène

7 Delosière $^{3}$, Fabrizio Ceciliani ${ }^{4}$, Sébastien Déjean ${ }^{6}$, Muriel Bonnet ${ }^{3 *}$

$8 *$ These authors are co-corresponding authors to this work.

9

\section{Affiliations:}

${ }^{1}$ Institute of Animal Science, Physiology Unit, University of Bonn, Bonn, Germany

$12{ }^{2}$ Research Institute for Farm Animal Biology (FBN), 18196 Dummerstorf, Germany

13 3INRAE, Université Clermont Auvergne, VetAgro Sup, UMR Herbivores, F-63122 Saint-Genès-Champanelle, France

$14 \quad{ }^{4}$ Department of Veterinary Medicine, Università degli Studi di Milano, Lodi, Italy

$15 \quad{ }^{5}$ BASF SE, 68623 Lampertheim, Germany

$16{ }^{6}$ Institut de Mathématiques de Toulouse, UMR5219, Université de Toulouse, CNRS, UPS, 31062 Toulouse, France.

17

18 Corresponding Authors:

1- Muriel Bonnet (muriel.bonnet@inrae.fr)

2- Harald M. Hammon (hammon@ fbn-dummerstorf.de) $1 \& 2$ are co-corresponding authors to this work.

\section{$23 \quad$ Highlights}

1- ANOVA-simultaneous component analysis applied to time course experimental design

2- Oxidation capacity as a signature of hepatic metabolic adaptation to lactation

3- Fatty acid (FA) supplementation amplified hepatic FA oxidation

27

4- Ligand-activated nuclear receptors primary regulate hepatic FA oxidation 


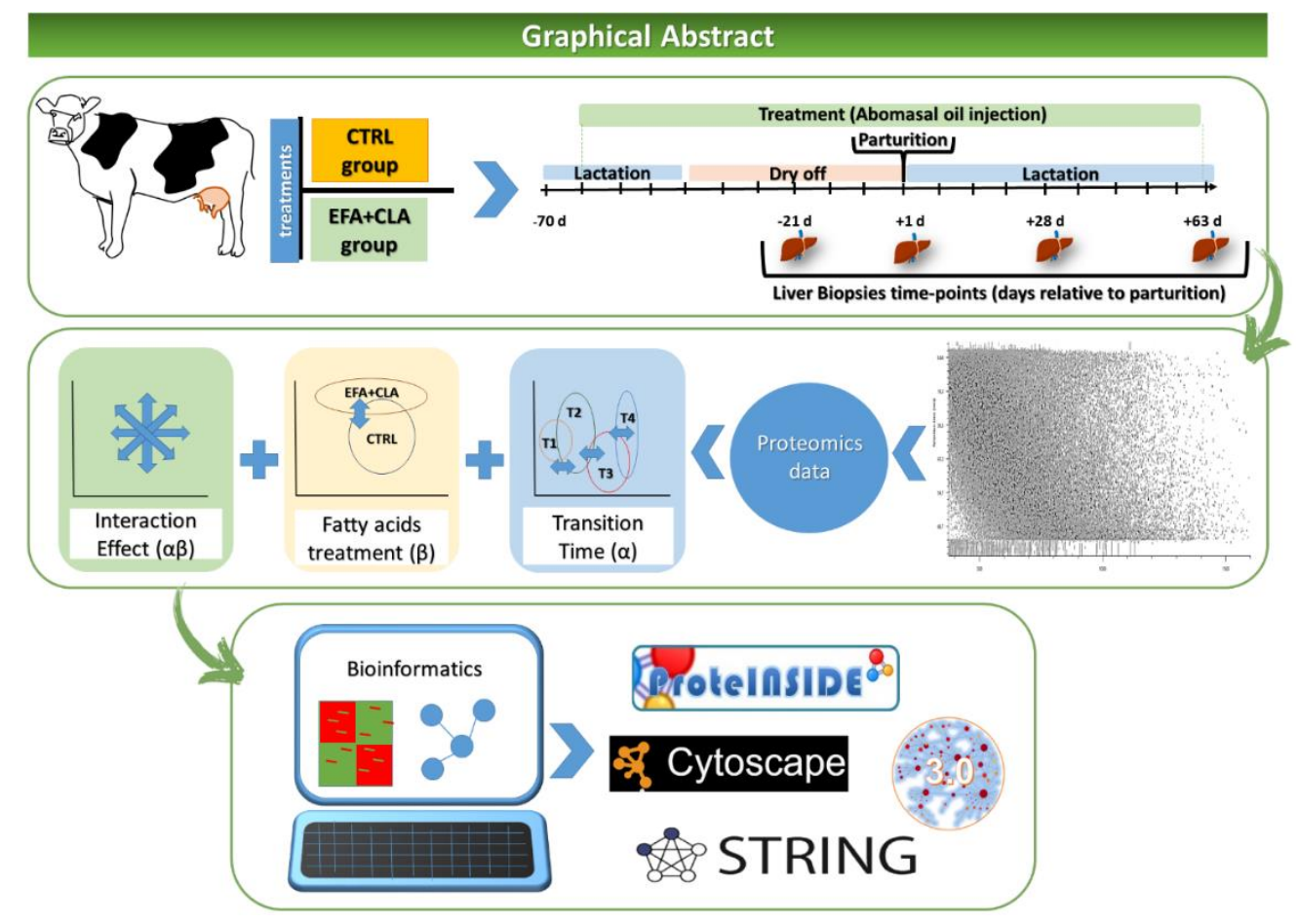

\section{Abstract}

32 Repeated measurements analysis of variance - simultaneous component analysis (ASCA) has been developed to handle complex longitudinal omics datasets and combine novel information with existing data. Herein, we aimed at applying ASCA to 64 liver proteomes collected at 4-time points (day $-21,+1,+28$, and +63 relative to parturition) from 16 Holstein cows treated from $9 \mathrm{wk}$ antepartum to $9 \mathrm{wk}$ postpartum (PP) with coconut oil (CTRL) or a mixture of essential fatty acids (EFA) and conjugated linoleic acid (CLA) (EFA+CLA). The ASCA modelled 116, 43, and 97 differentially abundant proteins (DAP) during the transition to lactation, between CTRL and EFA+CLA, and their interaction, respectively. Time-dependent DAP were annotated to pathways related to the metabolism of carbohydrates, FA, and amino acid in the PP period. The DAP between FA and the interaction effect were annotated to the metabolism of xenobiotics by cytochrome P450, drug metabolism - cytochrome P450, retinol metabolism, and steroid hormone biosynthesis. Collectively, ASCA provided novel information on molecular markers of metabolic adaptations and their interactions with EFA+CLA supplementation. Bioinformatics analysis suggested that supplemental EFA+CLA amplified hepatic FA oxidation; cytochrome P450 was enriched to maintain metabolic homeostasis by oxidation/detoxification of endogenous compounds and xenobiotics.

Keywords: ASCA; liver biopsy; LC-MS/MS; fatty acids; transition cows; cytochrome p450 


\section{Significance}

48 This report is among the first ones applying repeated measurement analysis of variance-simultaneous 49 component analysis (ASCA) to deal with longitudinal proteomics results. ASCA separately identified differentially 50 abundant proteins (DAP) in 'transition time', 'between fatty acid treatments', and 'their interaction'. We first 51 identified the molecular signature of hepatic metabolic adaptations during postpartum negative energy balance; the 52 enriched pathways were well-known pathways related to mobilizing fatty acids (FA) and amino acids to support 53 continuous energy production through fatty acid oxidation, TCA cycle, and gluconeogenesis. Some of the DAP were 54 not previously reported in transition dairy cows. Secondly, we provide novel information on the mechanisms by which 55 supplemented essential FA and conjugated linoleic acids interact with hepatic metabolism. In this regard, FA amplified

56 hepatic detoxifying and oxidation capacity through ligand activation of nuclear receptors. Finally, we briefly compared 57 the strengths and weaknesses of the ASCA model with PLS-DA and outlined why these methods are complementary. 


\section{Introduction}

59 A state of negative energy balance (NEB) during the transition from late gestation to early lactation initiates a series

60 of profound metabolic and physiological adaptations in dairy cows to meet the energy demands for milk production

61 [1]. During NEB, fatty acids (FA) are mobilised from adipose tissue to be oxidized in the liver for supplying energy

62 [2]. Therefore, the major adaptive mechanism is shifting towards the use of non-esterified fatty acids (NEFA) by 63 hepatocytes, where they are further metabolized via various pathways [3]. Numerous transcription factors and 64 coactivators, such as peroxisome proliferator-activated receptors (PPAR) and sterol regulatory elementbinding proteins (SREBPs), control these regulatory mechanisms [4]. Also, various nutritional treatments, e.g., FA that are not only substrates for generating energy but also natural ligands for nuclear receptors [5], may interact and impact metabolic adaptations [6, 7].

Over the past decades, mass spectrometry (MS)-based proteomics technology has emerged and matured as a powerful approach to discern the key factors contributing to systemic metabolic homeostasis and health in many species, including ruminants [8-11]. With a growing interest that has been paid to proteomics studies, it is not uncommon to have an intricate experimental design with different time points (or 'longitudinal'), treatments (multi-group, e.g. different dose groups), and multi-subject (containing data of multiple animals) [12]. For instance, in vivo longitudinal animal studies frequently deal with random physiological states (such as lactation, pregnancy, and growth), which could stand as the primary source of variation, especially if the treatment effect is negligible. Such intricate experimental designs call for specific multivariate analysis with predefined matrices of additive effects.

One approach would be the principal component analysis (PCA) which is designed for reducing the dimensionality of large datasets while increasing interpretability $[13,14]$. However, the straightforward use of PCA without predefining the factors may come up with clusters in which the primary sources of variation may be due to the longitudinal effect instead of the treatment effect [15]. Combining the analysis of variance with PCA led to the development of the ANOVA-simultaneous component analysis (ASCA) method (developed by Smilde [12]). This method is particularly suited for time-resolved studies and has the advantages of decomposing variability separately within the 'treatment' and 'time' and between 'time and treatment' (interaction of time and treatment). Subsequently, PCA is performed on each defined source of variation independently (for review [16]).

The ASCA design has been previously reported in some studies, including a metabolomics intervention study, in which guinea pigs were treated with varying doses of vitamin $\mathrm{C}$, and their urine metabolite profiles were analyzed using NMR spectroscopy at several points in time [12]. The application of this design is not limited to metabolomics [17], but there is no report on other omics-based datasets yet. linoleic acids (CLA) on the liver proteome of dairy cows in several time points from the ante (AP) to the postpartum (PP) period without considering time as a fixed effect (since it was not the main focus of our study [60]). This routine procedure had pointed out and emphasized on the treatment effect, and was complemented by our specific longitudinal 
from gestation to lactation could be particularly informative in understanding the physiology of adaptation and lactation as a secondary purpose of a study. Moreover, relatively little is known about hepatic metabolic adaptation in transition dairy cows in response to supplemented FA (interaction effect) at the proteome level.

In this study, we aimed at recruiting the repeated measures ACSA design to reuse our proteomics results and assess differentially abundant proteins (DAP) within the transition from gestation to lactation as an initial objective of this study. A further goal was to investigate how supplemented FA may interact with metabolic adaptations. To the best of our knowledge, this is the first report using the repeated measures ACSA on comprehensive untargeted longitudinal liver proteomics data set for interpreting the metabolic shifts related to FA supplementation in dairy cows during the transition period.

\section{Material and methods}

\subsection{Experimental design, sampling, and peptide preparation}

The study used raw LC-MS/MS results from our previously reported liver proteomics study [60]. Briefly, 16 multiparous Holstein dairy cows $(11,101 \pm 1,118 \mathrm{~kg}$ milk/305 d in second lactation and BW of $662 \pm 56 \mathrm{~kg}$; means \pm SD) were abomasally injected with either a control fat (coconut oil; CTRL, $\mathrm{n}=8$; Bio-Kokosöl \#665, Kräuterhaus Sanct Bernhard, KG, Bad Ditzenbach, Germany) or EFA+CLA supplement, containing a combination of linseed oil (DERBY® Leinöl \#4026921003087, DERBY Spezialfutter GmbH, Münster, Germany), safflower oil (GEFRO Distelöl, GEFRO Reformversand Frommlet KG, Memmingen, Germany) and Lutalin® (CLA, n = 8; 10 g/d cis-9, trans-11, trans- 10, cis-12 CLA, BASF SE, Ludwigshafen, Germany) from d 63 AP until d 63 post PP (Figure 1 A). The experimental procedures were carried out at the Research Institute for Farm Animal Biology (FBN), Dummerstorf, Germany and approved by German Animal Welfare Act (Landesamt für Landwirtschaft, Lebensmittelsicherheit und Fischerei Mecklenburg-Vorpommern, Germany; LALLF M-V/TSD/7221.3-1-038/15). Liver tissues were obtained from each animal on d -21 AP, and d 1, 28, (by biopsy) and 63 PP after slaughtering the cows (Figure 1 A). More information regarding diet ingredients, chemical composition, performance, and plasma metabolite data can be found elsewhere [18].

2.2. Liver sample preparation and proteomics analysis to in-gel digestion and the peptide mixtures were then analyzed using high-resolution nano-liquid chromatography (Ultimate 3000 RSLC nano-system (Dionex)) coupled to an Orbitrap Q Exactive HF-X mass spectrometer (Thermo Fisher Scientific) [60] (Figure 1 B). It is important to point out that some steps were considered to reduce betweengroup variability and increase the power of analysis. In this regard, LC-MS/MS was performed on all 64 samples consecutively but randomly without any order related to time or treatment using the same setting and unique analytical columns. Before and after MS analysis, LC-MS/MS efficiency (quality of liquid chromatography separation and mass 
spectrometry performance) was checked using the Pierce TM HeLa Protein Degradation Standard (catalogue number: 88328 Thermo Scientific ${ }^{\mathrm{TM}}$ ). For more details, see [60].

129

\subsection{Data processing}

Peptides MS/MS spectra were aligned to the reference sample automatically defined by Progenesis QI software (version 4.2, Nonlinear Dynamics, Newcastel upon Tyne, UK). It has to be highlighted that the reference sample is defined regardless of time or treatment, and alignment is done to all samples to obtain a set of comparable peaks. After peptide quantification, the identified/quantified peptide ions were searched against a Bos taurus decoy database (Uniprot, download date: 2019/11/07, a total of 37,513 entries) using MASCOT (version 2.5.1) interrogation engine. The specific validated peptides were then inferred to corresponding proteins and their intensities in Progenesis QI software (Figure 1B). A total of 1681 proteins were maintained for analysis after applying strict exclusion criteria (deamidated, carbamidomethyl, and oxidation contaminant proteins, having at least two peptides and two unique peptides, and presence in at least $50 \%$ of the samples in each treatment group/timepoint) [19].

\subsection{Decomposing matrices for ASCA and statistical analysis}

Statistical analysis was performed on the normalized log-transformed and auto-scaled (z-transformation) intensity values with the metaboAnalystR 3.0 package in $\mathrm{R}$ statistical software ( $\mathrm{R}$ version 4.0.0). The missing intensities were imputed and replaced with the small values (half of the smallest positive value in the dataset).

The ASCA method was described in detail previously $[12,20]$; here, we have briefly illustrated its design for our proteomics dataset. In this study, a balanced experiment was structured by ASCA. Our proteomics dataset comprised 64 distinct proteomes that were organized as described below (Figure 1C):

Individuals: 16 cows were included in the experiments.

$(\alpha)$ Time: four timepoints days $-21,+1,+28$, and +63 relative to parturition were considered as time variable (16 individual* 4 timepoints).

( $\beta$ ) Treatment: The two treatment groups, including control and EFA+CLA, were inputted into the model as treatment variables (32 individuals $* 2$ treatment groups)

$(\alpha \beta)$ Interaction of time and treatment: possible mixtures $=8$ individuals $* 2$ treatment groups $* 4$ timepoints.

The first step was to perform a two-way repeated-measures Analysis of variance (ANOVA) on each variable described above individually, according to equation 1 ,

$$
\text { (1) } x i j k p=\mu+\alpha i+\beta j+(\alpha \beta) i j++S k(j)+\varepsilon k i j \text {. }
$$

Equation (1) indicates a series of $\mathrm{j}$ ANOVAs where $\mu$ is an overall offset, $\alpha \mathrm{i}$ the effect of the first factor (transition from gestation to lactation), $\beta \mathrm{j}$ the effect of treatment (supplemented FA), $(\alpha \beta) \mathrm{ij}$ the interaction between them, $\operatorname{Sk}(\mathrm{j})$ is the random effect of the $\mathrm{k}^{\text {th }}$ subject and ekij the residuals. 
164 Then, applying PCA to each score sub-matrix in (1) (indicated by $\mathrm{T} \alpha, \mathrm{T} \beta$, and $\mathrm{T} \alpha \beta$ ) and submodel loadings (are given by matrices $\mathrm{P} \alpha, \mathrm{P} \beta$, and $\mathrm{P} \alpha \beta$ ) and examining estimated effects for all variables simultaneously by

(2) $\mathrm{X}=\mathrm{Xm}+\mathrm{TaPaT}+\mathrm{TbPbT}+\mathrm{TabPabT}+\mathrm{Xe}$,

where $\mathrm{T}$ and $\mathrm{P}$ - as mentioned before- represent the scores and loadings matrices for each corresponding factor or interaction, respectively, and Xe defines the residual or deviations of each individual replicate from the average effects. The performed operation (2) was a Simultaneous Component Analysis (given the name ASCA) or repeated PCA on a common set of measured variables allocated to predefined matrices. The following criteria were set to decompose the ASCA model in R: Leverage threshold=0.9, and alpha threshold $=0.05$.

173

\subsection{Bioinformatics analysis of differentially abundant proteins}

175 Before bioinformatics analysis, proteins' accession was converted into Gene ID by the UniProt (retrieve/ID mapping) 176 database conversion tool, and undefined proteins were blasted and replaced with their Gene ID in other species. The 177 Gene Ontology (GO) and Kyoto Encyclopedia of Genes and Genomes (KEGG) pathways enrichment analysis of DAP 178 were performed with STRING (version 11.0) and proteINSIDE (version 1.0) setting B. Taurus genome as background, 179 and only pathways enriched with P-value $<0.05$, corrected to FDR with Benjamini-Hochberg method (p-adjust < 180 0.05) and at least two hits in each term were considered significantly enriched (Figure $1 \mathrm{C}$ ). Protein-protein interaction 181 networks were constructed and visualized by inputting the DAP identified on main effects (time, treatments, and their 182 interaction) to Cytoscape version 3.8.2 software, in which nodes and edges represent proteins and their interactions, 183 respectively [19]. 


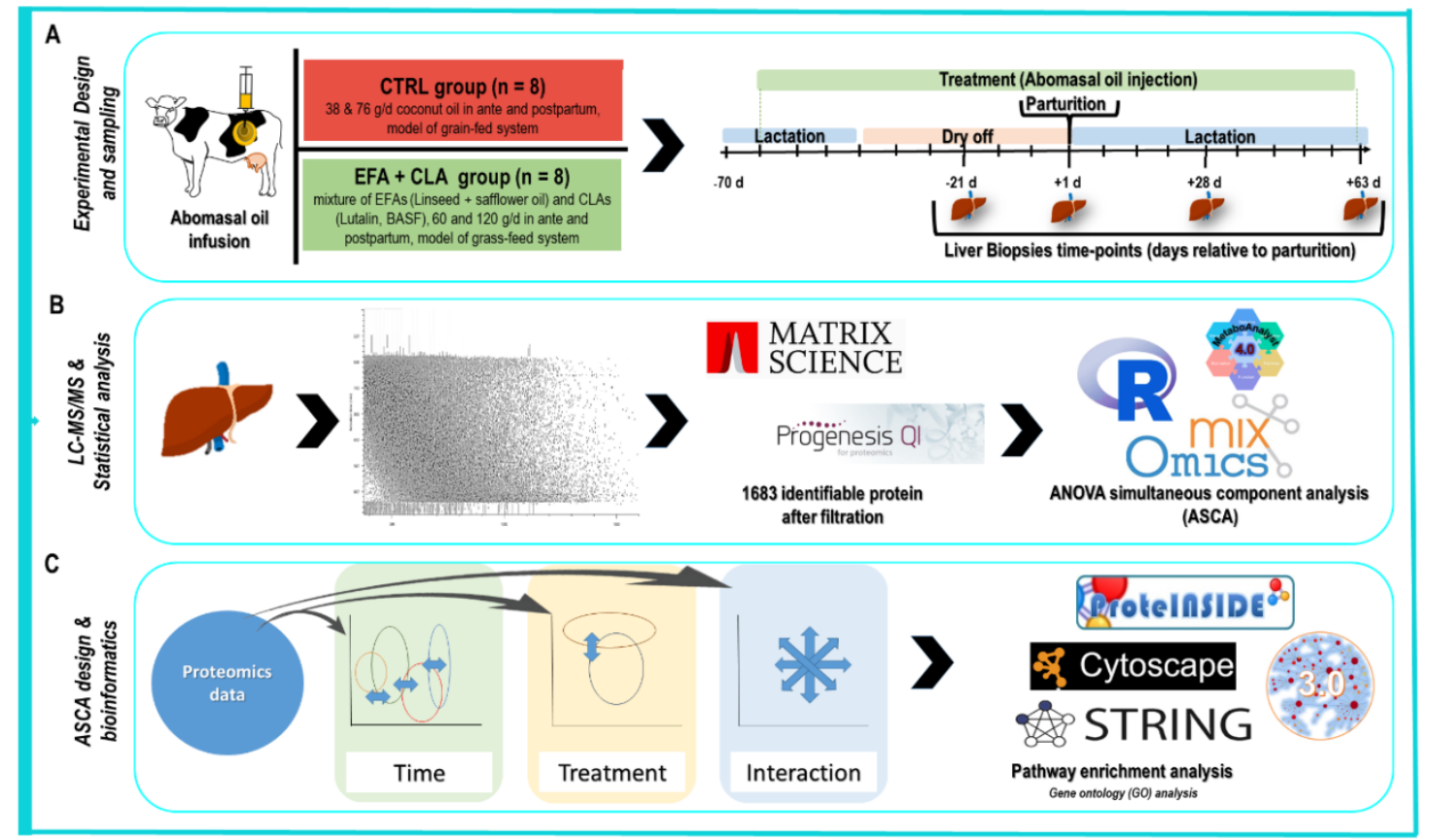

Figure 1) Schematic diagram of the (A) study design, (B) proteomics workflow and peptide identification, and (C) statistical analysis and bioinformatics pipeline. (A) Timeline of supplementation (from $-63 \mathrm{~d}$ ante to $+63 \mathrm{~d}$ postpartum) and liver biopsy collection $(-21 \mathrm{~d},+1 \mathrm{~d},+28 \mathrm{~d}$, and $+63 \mathrm{~d}$ relative to parturition). Bold lines indicate liver biopsy sampling timepoints. (B) High-resolution LC-MS/MS analysis, peptide alignment (progenesis), and protein identification (mascot) procedure were performed by Progenesis software coupled with the Mascot search engine, statistical analysis was based on Multivariate Analysis of variance - simultaneous component analysis (ASCA), and (C) ASCA design and bioinformatics analysis.

\section{Results and discussion}

\subsection{Differential proteomic analysis: repeated measurements analysis of variance - simultaneous component analysis}

From a total of 1681 proteins, 116 proteins during the transition period, 43 proteins between treatments, and 97 proteins in the interaction of them were identified as differentially abundant (Table 1, more details are provided [19]). Figure 2 represents the major pattern described by the ASCA model associated with transition time, FA treatment, and their interaction, respectively. Figure $2 \mathrm{~A}$, is a time score plot based on component 1 (52.24\% of variation explained) and demonstrated that there is a considerable difference (elbow break) between days 0 and 28. Figure $2 \mathrm{~B}$ showed that the groups differed in their principal component (PC) 1 scores (100\% of variation explained), with the CTRL and EFA+CLA groups exhibiting the lowest and highest scores, respectively. Figure $2 \mathrm{C}$ visualizes the major pattern assessed for the interaction effect on PC1 (more than 50\% of variation explained) and PC2 (more than $30 \%$ of variation explained). Leverage/SPE scatter plots, scree plots, and the permutation tests are provided in Supplementary Figure S1. 
A)
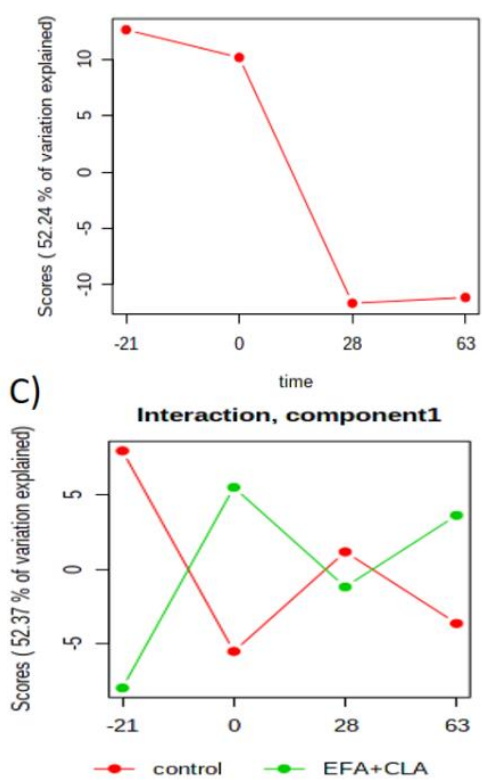

- control $\rightarrow$ EFA+CLA

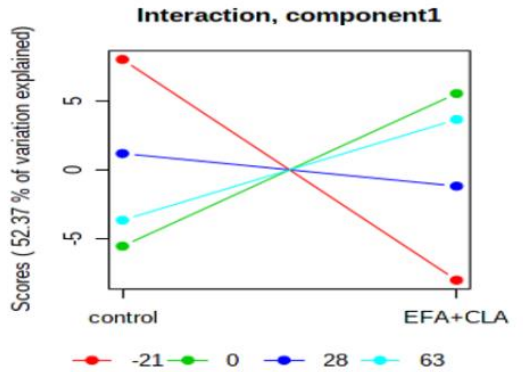

B)

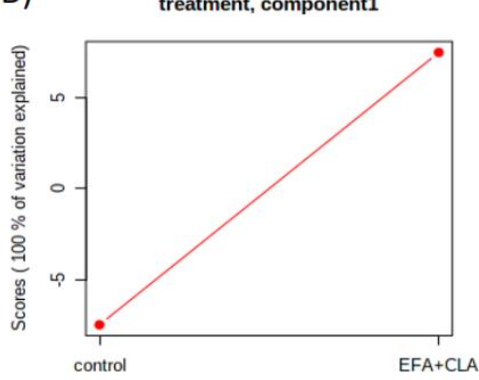

treatment

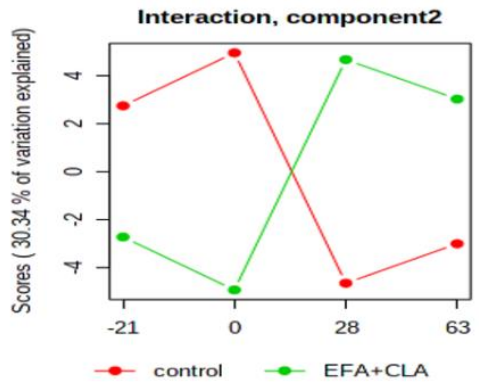

- control $\rightarrow$ EFA+CLA

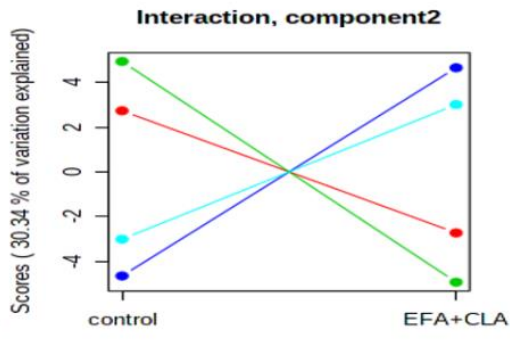

$\rightarrow-21 \rightarrow 0 \rightarrow 28 \rightarrow 63$

Figure 2) Major patterns associated with transition time (A), FA treatment (B) and their interaction (C) calculated by analysis of variance simultaneous component analysis (ASCA), in dairy cows supplemented with or without EFA+CLA in 4 time-points $(-21,+1,+28$, and $+63 \mathrm{~d}$ relative to parturition. The $\mathrm{x}$-axis indicates the scores and the y axis indicates the variables (different timepoints (a), CTRL and EFA+CLA (b), and interaction of them $(a b)$.

\subsection{Gene ontology and functional enrichment analyses of differentially abundant proteins during the} transition period

The relative abundance of DAP during the transition period is illustrated in a Heatmap (fold changes ranged from -4 to +4 ) in Figure 3. The protein abundance patterns within time points are graphed in the score plot (Figure 2 a), in

217 which the only considerable difference among them was observed between $d+1$ and $d+28$, that was also seen by two separate clusters containing d -21 AP and $+1 \mathrm{PP}$ as the first cluster (AP-cluster1) and d +28 and $+63 \mathrm{PP}$ as the second one (PP-cluster2). The first cluster (AP-cluster1) was representative of AP; vice versa, PP-cluster2 represented the PP period. Out of the 116 DAP obtained during the transition period, the relative abundance of 93 proteins increased, and 23 proteins decreased in PP-cluster2 compared with AP-cluster1 [19]. 


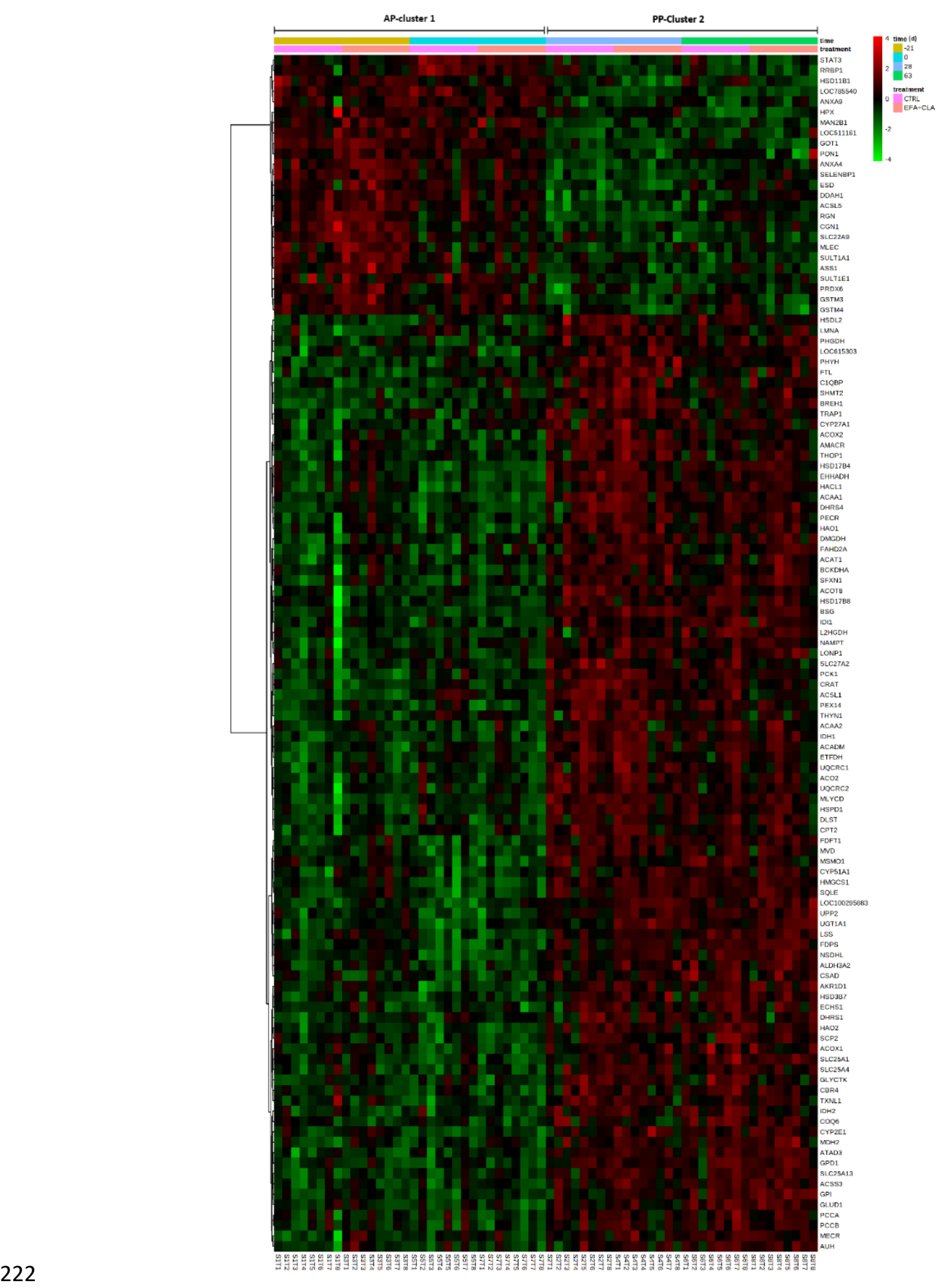

223 Figure 3) Hierarchical clustering and heatmap representation of differentially abundant proteins during the transition from late gestation to lactation 224 in dairy cows. Rows are respectively sorted by similarity as indicated by the left (proteins) dendrograms. Red and green represent increased and 225 decreased protein abundance, respectively. The colour code for different timepoints and treatments is provided on the right-hand side. 
Overabundant proteins (containing phosphoenolpyruvate carboxykinase (GTP) (PCK1), hydroxy acid oxidase 2 (HAO2), 3-hydroxy-3-methylglutaryl coenzyme A synthase (HMGCS1), isocitrate dehydrogenase [NADP] (IDH1), solute carrier family 25 member 13 (SLC25A13), squalene monooxygenase (SQLE), acetyl-CoA acyltransferase 1 (ACAA1), Dihydrolipoamide S-Succinyltransferase (DLST), Acyl-CoA Thioesterase 2(ACOT2), hydroxysteroid (17beta) dehydrogenase 4 (HSD17B4), isocitrate dehydrogenase [NADP], mitochondrial (IDH2), peroxisomal trans-2enoyl-CoA reductase (PECR), acyl-CoA synthetase long-chain family member 1 (ACSL1), carnitine Opalmitoyltransferase 2, mitochondrial (CPT2), and cytochrome P450 enzymes (CYP2E1, CYP51A1, and CYP27A1)) were annotated by 98 enriched GO terms in the biological processes (BP) category. They were mainly related to the metabolic processes of energy-related substrates such as carbohydrates, amino acids (AA), lipid and FA, phospholipid, acetyl-CoA, organic cyclic compound, ketone, and carboxylic acid (complete list in [19]). Underbundant proteins including peroxiredoxin-6 (PRDX6), glutathione S-transferase Mu 3 \& Mu 4 (GSTM3 \& GSTM4), and ASCL5 were annotated by BP GO terms to be related to carbohydrate metabolic process, chemical and ion homeostasis, and glutamine family AA catabolic process (complete list provided in [19]). Moreover, the functional analysis highlighted the enrichment of 46 KEGG pathways, including peroxisome, FA metabolism, valine, leucine and isoleucine degradation, PPAR signalling pathway, primary bile acid (BA) biosynthesis, steroid biosynthesis, citrate cycle (TCA cycle), biosynthesis of AA, metabolism of xenobiotics by cytochrome P450, pyruvate metabolism, biosynthesis of unsaturated FAs, pentose phosphate pathway, synthesis and degradation of ketone bodies, glycolysis/gluconeogenesis, and arachidonic acid metabolism in PP-cluster2 (Figure 4).

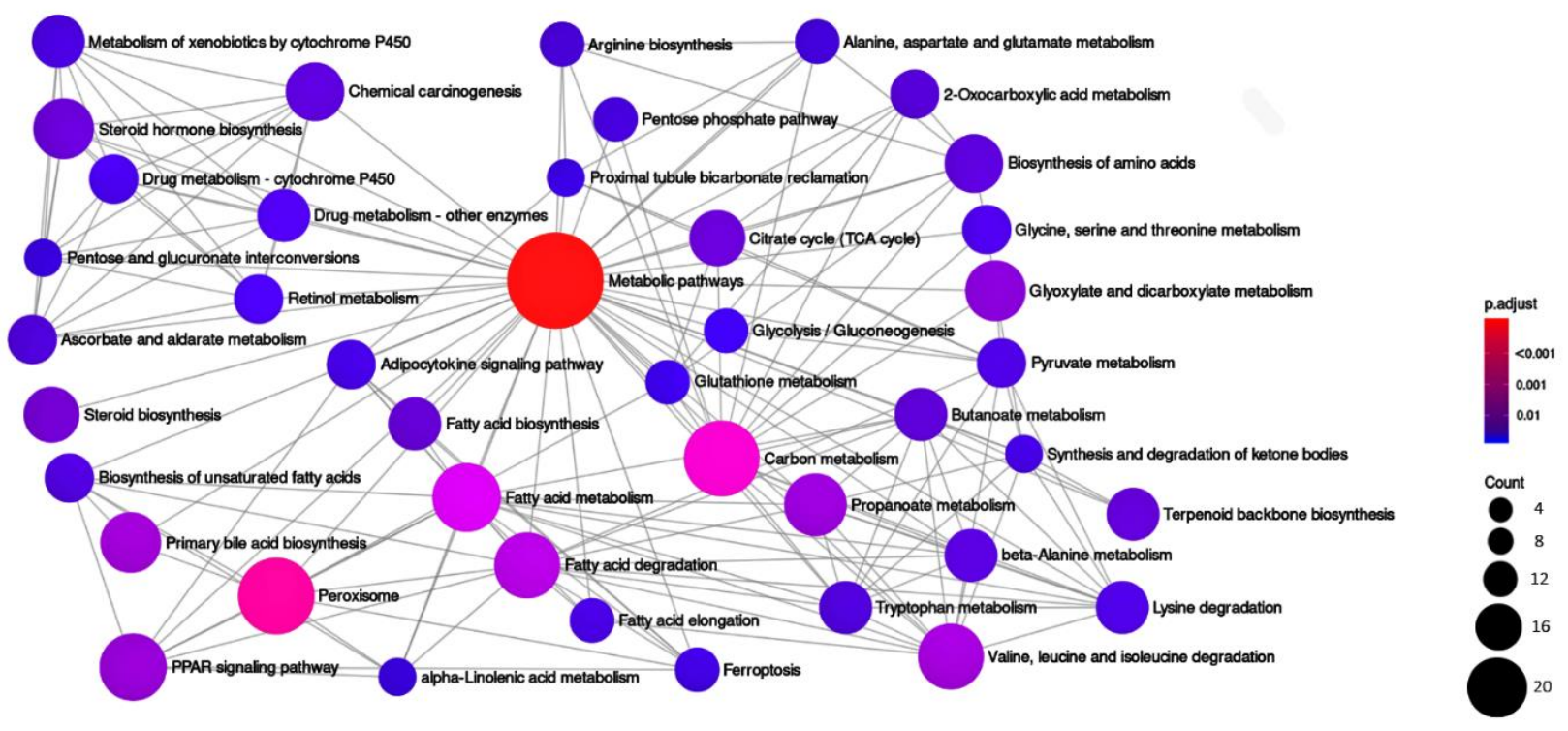

Figure 4) Kyoto Encyclopedia of Genes and Genomes (KEGG) pathway enrichment analysis of differentially abundant proteins (DAP) during the transition from late gestation to lactation in dairy cows. The colour of the nodes represents the $-\log 10$ (adjusted P-value); Node size represents the number of DAP contained in the node (smaller indicates lesser DAP, bigger indicates more DAP). 
These findings were consistent with previous liver proteome [21], and transcriptome [22] studies that have reported enrichment of carbohydrates, lipids, and protein metabolism-related pathways in the early and/or peak of lactation compared to the dry period to support milk synthesis. Since none of the cows in any treatment group showed any signs of metabolic disorders, all these massively enriched pathways could be considered as conventional metabolic adaptations to preserve whole-body metabolic homeostasis during the NEB period. Indeed, we have previously reported [18] the elevated plasma concentrations of NEFA and $\beta$-hydroxybutyrate (BHB) during the transition from late pregnancy to early lactation, implying that dairy cows from the present study were in a classical physiological NEB state. Thus, within the liver increased gluconeogenesis and ketogenesis are expected to interconvert and metabolize nutrients to support pregnancy and lactation. Consistent with this very general view of liver metabolic adaptations, we have identified proteins involved in ketogenesis, gluconeogenesis, and oxidative capacity through both the TCA cycle and the cytosolic organelles synthesis. Indeed, once taken up by the liver, NEFA are oxidized either via Acetyl-CoA through the TCA cycle or in ketone bodies from ketogenesis. The over-abundance of ACO2, DLST, IDH1, IDH2, MDH2, and PCK1 related to the TCA cycle, as well as the over-abundance of ACAT1, and HMGCS1 involved in ketogenesis, in the PP period relative to the AP period, strengthened the robustness of the proteome analysis and the ASCA analysis. Indeed most of them were previously identified by differential proteome during the transition period [11, 21, 23-25]. Some proteins may be highlighted such as the overabundance of both the ACO2 and IDH mitochondrial enzymes known to induce $\alpha$ ketoglutarate (AKG) production (from citrate) that serves as an energy source and also as a precursor for glutamine, gluconeogenesis, and synthesis of acute-phase proteins [26]. We observed an overabundance of both cytosolic and mitochondrial IDH isozymes (IDH1 and IDH2, respectively) in the PP period, indicating activated IDH2/IDH1 shuttle transferring high energy electrons in the form of NADPH from mitochondria to cytosol [27]. Moreover, an overabundance of ACAT1 and HMGCS1 was reported in feed-restricted ketotic cows in the PP period [28]. Part of the well-known DAP involved in FA oxidation and mevalonate pathway were highlighted in the schematic Figure 5.

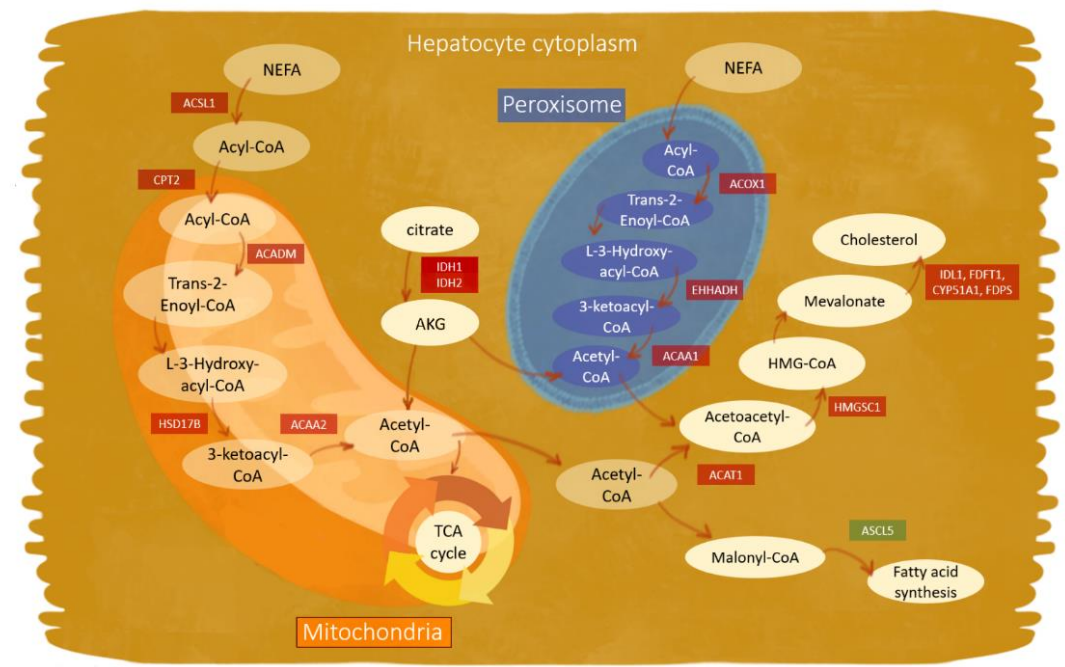
postpartum period are highlighted; red colour indicates upregulation; green designated downregulation. 
277 In dairy cows, propionate as a primary source but also lactate, AA (specifically L-alanine), and glycerol can be 278 oxidized indirectly through the TCA cycle to supply carbon for gluconeogenesis. The entry point of these substrates 279 differs and could be through either succinate, oxaloacetate (OAA), or Acetyl-CoA, which is under the control of 280 different isoforms of phosphoenolpyruvate carboxykinase (PEPCK). Here, we observed the PP overabundance of 281 PCK1 (cytosolic form) enzyme, which is a rate-limiting enzyme in gluconeogenesis [29], controlling the entry from AA and propionate [30]. In line with our results, it has been reported that the expression of PCK1 is elevated with increasing feed intake during early lactation [31,32].

284 The oxidative capacity of the TCA cycle is dependent on the supply of OAA (carbon carrier) from pyruvate by the action of pyruvate carboxylase (PC) to maintain a 1:1 relationship between OAA and acetyl-CoA [33]. The results revealed an overexpression trend (fold change $=1.65$ ) of the PC enzyme, although its expression was not modeled as differentially abundant. It is critical to balance the synthesis of metabolic intermediates (anaplerosis) and the extraction of metabolic intermediates for breakdown (cataplerosis), especially during the transition period to fuel gluconeogenesis and maintaining carbon homeostasis [33]. Therefore, it can be concluded that the overabundance of both PC and PCK1 probably concur to increase the gluconeogenesis capacity while keeping the balance between anaplerosis and cataplerosis.

292 Moreover, we observed an enrichment of the peroxisome proliferator-activated receptors (PPAR) pathway, which is 293 known to have a pivotal role in cycling lipid and carbohydrate substrates into glycolytic/gluconeogenic pathways favoring energy production [34]. Accordingly, an overabundance of Acyl-CoA dehydrogenase (ACADM) which is involved in PPAR signaling and carbon and FA metabolism, combined with the overabundance of long FA transporter (SLC25A1 and SLC25A13) in the PP period, suggest a higher transport activity of FA from the plasma into the hepatocytes, thus supporting a higher level of FA $\alpha$ and $\beta$-oxidation for energy supply. In the PPAR pathway, the relative abundance of Enoyl-CoA Hydratase and 3-Hydroxyacyl CoA Dehydrogenase (EHHADH) along with EnoylCoA Hydratase, Short Chain 1 (ECHS1) was increased; both proteins have been previously reported to be involved in milk FA metabolism in humans [35] and cow [36] studies, not only through the PPAR but also through AMPK (5' AMP-activated protein kinase) signaling pathways. The significant effects of ECHS1 on long-chain unsaturated, medium-chain saturated FA, and milk FA traits in dairy cattle were discussed elsewhere [36]. The enrichment of the PPAR pathway is also in line with the repeatedly reported role of PPARs as a sensor of NEFA levels [37, 38]. Besides, PPAR are also involved in transcriptional regulatory mechanisms coordinating the abundance and enzyme content of organelles [39]. In this regard, we observed the enrichment of pathways related to organelles, in particular, peroxisomes and mitochondria in PP-cluster2, with more than 20 DAP in the peroxisome, including IDH, Acyl-CoA dehydrogenases (ACADs), Sterol Carrier Protein 2 (SPC2), and ACADM. Both peroxisomes and mitochondria are remarkably dynamic adapting their number and activity depending on the prevailing environmental conditions i.e., excessive NEFA can thus be used directly as substrate and indirectly through PPAR activation [39]. Along with mitochondria, peroxisomes play a crucial role in cellular lipid hemostasis, in which the overabundance of SPC2 indicates activation of the peroxisomal cholesterol transport from the cytoplasm and an induced FA $\beta$-oxidation [40]. Moreover, AA metabolism, including glycine, serine, isoleucine, threonine, and tryptophan metabolism, was enriched in synchronized with mobilizing skeletal muscle protein during the PP NEB period. The released AA were primarily 
not metabolized in the liver to support mammary glands' milk protein synthesis [41, 42]. Considering the differences between the AA profile of muscle and milk [43, 44], the enrichment of various AA metabolism was probably a counter-regulation to maintain the AA ratio, precisely because AA are only available in limited quantities. Interestingly, we observed the degradation of the branched-chain AA (BCAA, i.e., valine, leucine, and isoleucine) among the most significantly enriched pathways in the PP-cluster2. In this regard, BCAA, in contrast to other AA, are less degraded in the liver (first-pass hepatic catabolism) and are preferentially metabolized in extrahepatic tissues [45, 46]. Activated hepatic degradation of BCAA, in particular during the transition period may indicate that they primarily converted to other AA or fed into TCA cycle/ketogenesis pathways. The present results suggest a strong relationship between ketogenesis and BCAAs, accordingly to what was previously reported [11], in such a way that when citrate synthesis (intensively driven by BCAA degradation but also FA oxidation) exceed the TCA capacity, its surplus is directed to ketogenesis. In this pathway, 11 DAP were involved among which ECHS1, EHHADH, ACAA, and ACADM were discussed previously. Here, the overabundance of the $\alpha$ and $\beta$ subunits of the propionyl-CoA carboxylase enzyme (PCCA and PCCB) that catalyzes the conversion of propionyl-CoA to methylmalonyl-CoA, revealed an activated gluconeogenesis pathway using propionate as a substrate, and thus feeds the TCA cycle with limiting intermediates. Proteomic results provided an in-depth overview of metabolic adaptations during the NEB period. To summarize, FA metabolism and degradation, PPAR signaling pathway, peroxisome, and TCA cycle were enriched to enhance lipid and carbohydrate catabolic processes that fuel glycolytic/gluconeogenic pathways favoring energy production rather than storage. Also, the enrichment of pathways related to FA biosynthesis, elongation, and biosynthesis of unsaturated FA, along with $\alpha$-linolenic acid metabolism, suggest that the identified proteins are involved in providing intermediates/backbones to be used later by the mammary gland for milk fat synthesis. Furthermore, metabolic adaptations were initiated in response to NEB by mobilizing energy substrate to fuel the TCA cycle with OAA, succinate, and $\alpha$-ketoglutarate, by activating a broad range of pathways related to carbohydrate, lipid, AA, and energy metabolism.

\subsection{Gene ontology and functional enrichment analyses of differentially abundant proteins between treatment groups}

We have previously reported in detail proteins and their associated pathways affected by FA supplementation at several timepoints around parturition [60]. Here, we pooled all timepoints and reported the enriched pathways affected by EFA+CLA treatment (regardless of time). Of the 43 DAP modeled within the treatment, 31 proteins had higher, and 12 proteins had lower abundance in EFA+CLA with a fold change ranging from -3 to 3 (Figure 6).

Pathway and gene ontology analyses revealed that overabundant proteins were annotated by 28 enriched GO terms within the BP category, including carboxylic acid biosynthetic process (GO:0046394) and metabolic process

348 (GO:0044237), NADP metabolic process (GO:0006739), oxidoreduction coenzyme metabolic process 349 (GO:0006733), coenzyme (GO:0006732) and cofactor (GO:0051186) metabolic process, and carbohydrate catabolic process (GO:0016052). Underabundant proteins did not annotate to any pathways. 


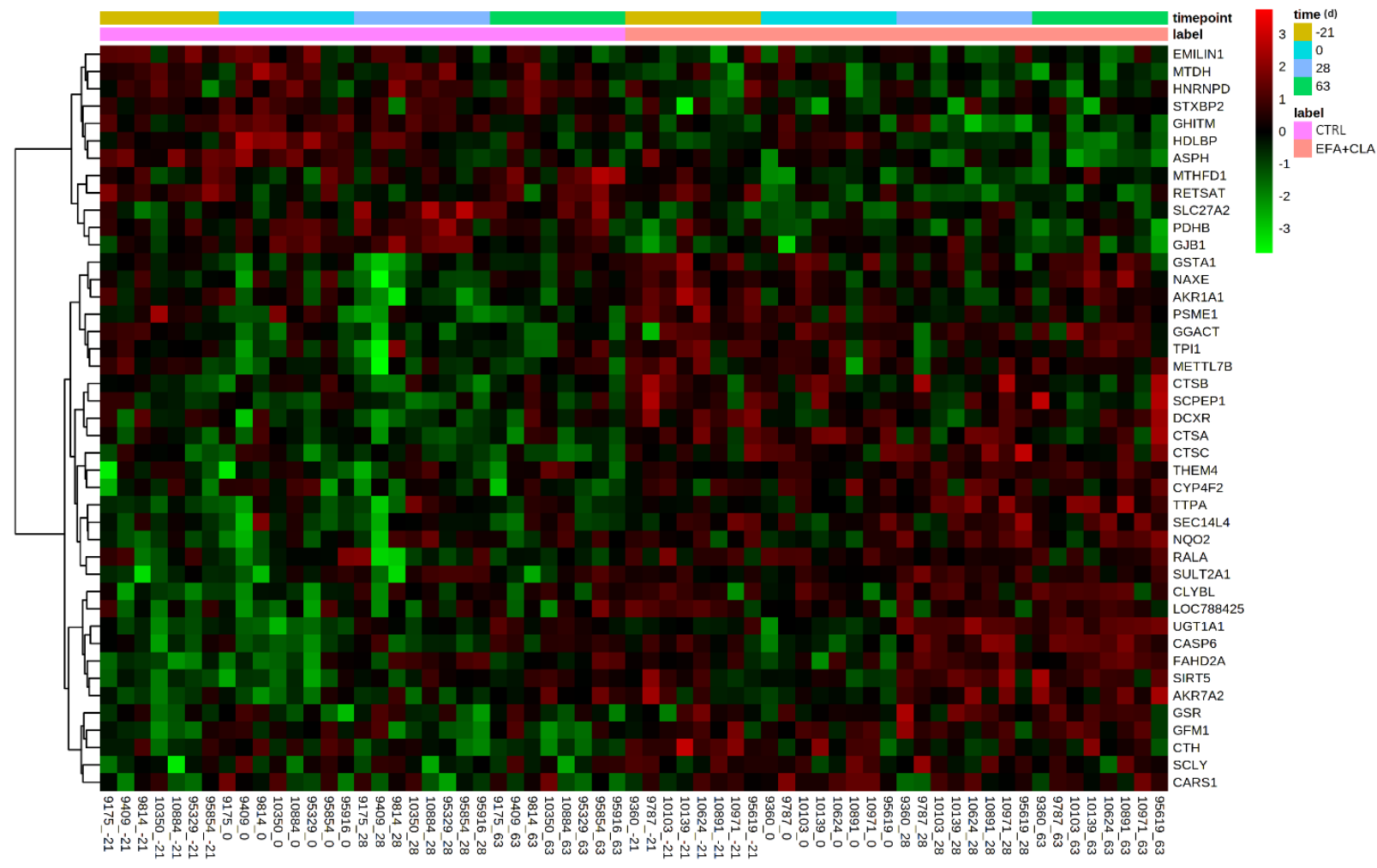

Figure 6) Hierarchical clustering and heatmap presentation of differentially abundant proteins between CTRL and EFA+CLA. Rows are respectively sorted by similarity as indicated by the left (proteins) dendrograms. Red and green represent increased and decreased proteins abundance, respectively. The colour code for different timepoints and treatments is provided on the right-hand side.

Moreover, 11 KEGG pathways were found to be enriched when the 43 DAP were considered: metabolism of xenobiotics by cytochrome P450, pentose and glucuronate interconversions, glycolysis/gluconeogenesis, lysosome, apoptosis, glutathione metabolism, retinol metabolism, chemical carcinogenesis, drug metabolism - cytochrome P450, and drug metabolism - other enzymes (Figure 7).

360 The most significantly enriched KEGG pathway was the metabolism of xenobiotics by cytochrome P450 with four

361 DAP, including an overabundance of glutathione S-transferase A1 (GSTA1), aldo_ket_red domain-containing protein (AKR7A2), sulfotransferase family 2A member 1 (SULT2A1), UDP-glucuronosyltransferase family 1 member A1 (UGT1A1). Cytochrome P450 (CYP) pathways constitute a superfamily of more than 1000 enzymes containing heme, capable of affecting various metabolic and biosynthetic processes by oxidizing different structural compounds, including steroids, prostaglandins, FA, derivatives of retinoic acid, and xenobiotics [47, 48]. For instance, the involvement of CYP enzymes in the hepatic biotransformation of cholesterol, its degradation to bile acids (BA), detoxification, and metabolic homeostasis has been the subject of many research studies [49, 50]. We have previously reported the involvement of specific CYP enzymes in different time points during the transition period that could be time-dependent or related to the fluctuating concentration of FA (NEFA and supplemented FA) serving as specific substrates [60]. Different CYP enzymes are capable of catalyzing the oxidative biotransformation of FA which is

371 known as hepatic $\omega$-oxidation of FA and functions primarily to facilitate their elimination when mitochondrial $\beta$ - 


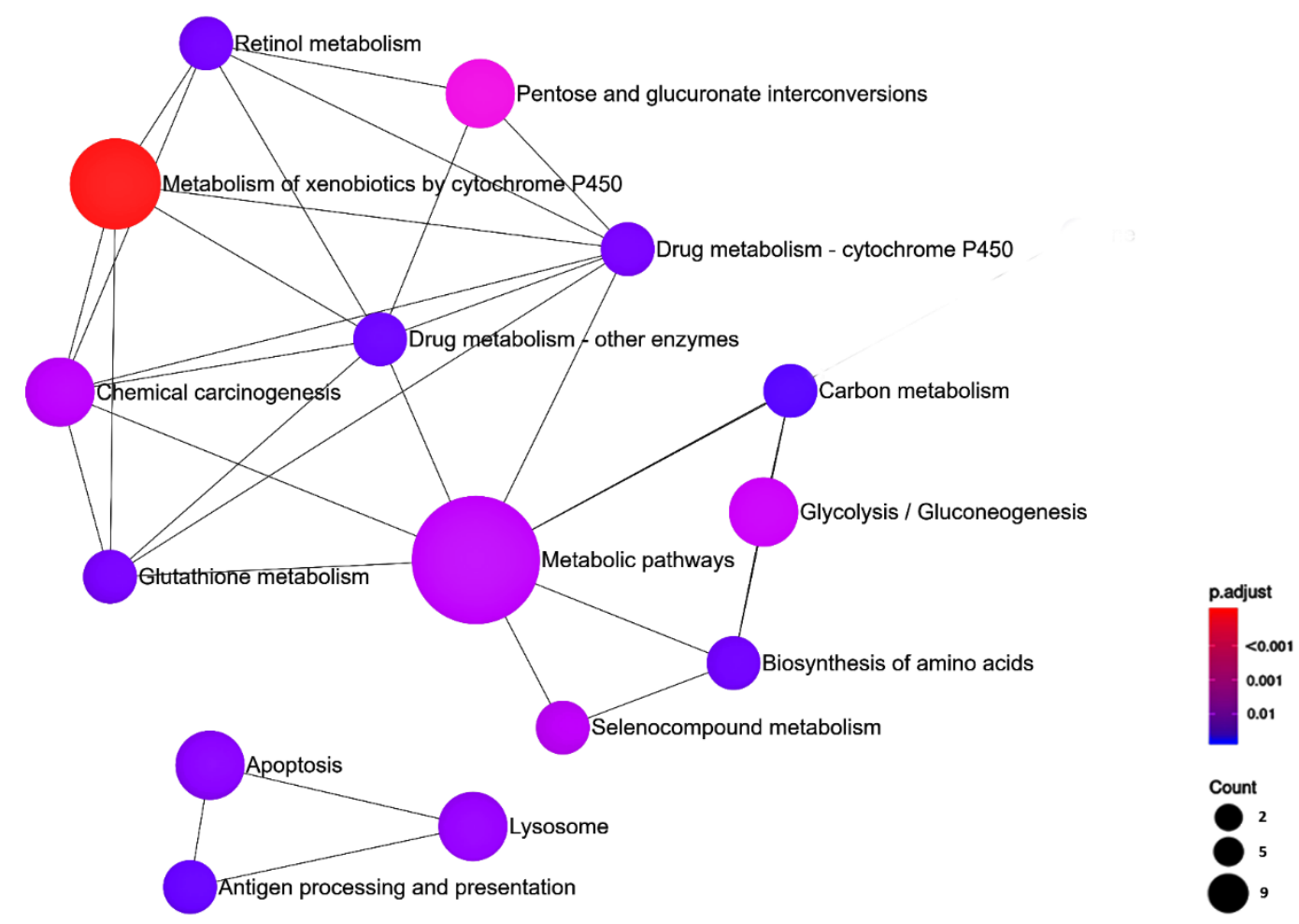

Figure 7) Kyoto Encyclopedia of Genes and Genomes (KEGG) pathway enrichment analysis of differentially abundant proteins (DAP) between CTRL and EFA+CLA. The colour of the dots represents the -log 10 (adjusted P-value); the size of the dots represents the number of DAP in the pathway.

Another mechanism that regulates CYPs expression is through the activation of the PPAR pathway [51]. It has been shown that PUFA, especially $\omega-3$ FA, compete with NEFA for ligand activation of PPAR [37], suggesting a potential role of these receptors in drug metabolism as well as metabolic homeostasis related to FA metabolism. All these pieces of evidence imply that the cytochrome P450 system may play a key role in regulating hepatic lipid homeostasis, as proposed earlier [49]. Another study indicated the involvement of CYP genes in steroidogenesis converting cholesterol to pregnenolone and consequently to dehydroepiandrosterone [52]. In this study, the steroid hormone biosynthesis pathway was enriched by the overabundance of the LOC100138004 protein. Steroid biosynthesis mainly occurs in the gonads and the adrenal glands, while the liver is considered a site for steroid hormone inactivation [53]. Recent observations in dairy cows have shown that providing a gluconeogenic feed (propylene glycol) or treatment with insulin infusion decreased the hepatic expression of CYP enzymes (CYP2C and CYP3A activity) responsible for hepatic progesterone catabolism, which could result in early fetal losses [54]. In the current study, neither the concentration of insulin [55] nor the hepatic abundance of CYP2C and CYP3A enzymes were affected by the treatment. Hence, identified CYP enzymes 
were time-specific; they were not presented at all time points to be considered DAPs with the repeated measurement ASCA model. Thus, the ASCA method has identified additional proteins with the CYP pathways that exemplify first the benefit of combining ASCA and PLS-DA analysis, and second the centrality of CYP pathways in responses to EFA+CLA supplementation in dairy cows.

The enrichment of glutathione metabolism indicates a role in the maintenance and regulation of the thiol-redox status against generated ROS during the CYP catalytic cycle. As previously discussed, an elevated rate of peroxisomal and mitochondrial FA oxidation in dairy cows during early lactation is accompanied by greater oxidative production, which may be counteracted by activation of the anti-oxidative machinery system in the liver. Within this pathway, the abundances of two key enzymes, glutamate-cysteine ligase catalytic subunit (GCLC), which is a rate-limiting enzyme in glutathione metabolism, and glutathione reductase (GSR) that converts oxidized GSH to the reduced form were elevated. Associated with the glutathione and cytochrome metabolism pathway, GSTM 3 and 4 both belonging to the glutathione S-transferase (GST) superfamily, were downregulated. Members of the GST family are upregulated in response to oxidative stress and are involved in catalyzing the xenobiotic-derived electrophilic metabolites, in steroid hormone biosynthesis, in eicosanoid metabolism and, and in MAPK pathway (for review, see [56]). Moreover, PRDX6, a member of the peroxiredoxin antioxidant enzymes family, is involved in the detoxification process against oxidative stress through glutathione peroxidase. In this regard, Abuelo et al. [57] reported a gradual increase in oxidative stress status after calving due to fat mobilization. Due to the higher $\omega$-oxidation capacity in EFA+CLA supplemented cows [60], it seems conceivable to activate GSH synthesis for avoiding oxidative stress. Collectively, the results indicated that EFA+CLA supplementation enriched cytochrome P450 as a core affected pathway. It is worth mentioning that identifying DAP between CTRL and EFA+CLA group in each timepoint [60] provided partially different patterns (only a few proteins in common) in comparison to identifying DAP between pooled CTRL and EFA+CLA group (without considering time). This is because we observed a time-specific pattern for DAP, which would not be detectable by the ASCA model. The ASCA would only consider a protein as DAP if it had a constantly lower/higher abundance in all timepoints. Interestingly, metabolism of xenobiotics by cytochrome P450, drug metabolism - cytochrome P450, drug metabolism - other enzymes, and retinol metabolism were enriched as the main affected pathways by both methods.

\subsection{Gene ontology and functional enrichment analyses of differentially abundant proteins within the interaction of transition period and fatty acid supplementation}

Herein, 97 proteins were found to be affected by the interaction of time and FA supplementation (with a fold change ranging from -6 to +6$)$; proteins were fluctuating between two independent parameters $\left(\alpha^{*} \beta\right)$ and therefore reporting the individual over- or under-abundancy for each protein is not feasible. The relative abundance of proteins modelled in the interaction effect is graphically presented in a Heatmap (Figure 8). The GO enrichment analysis revealed that these proteins were annotated by 65 enriched GO terms within the BP category such as cellular process (GO:0009987), organonitrogen compound metabolic process (GO:1901564), protein metabolic process (GO:0019538), peptide 
biosynthetic process (GO:0043043), gene expression (GO:0010467), translation (GO:0006412), electron transport chain (GO:0022900), and response to stress (GO:0006950) [19].

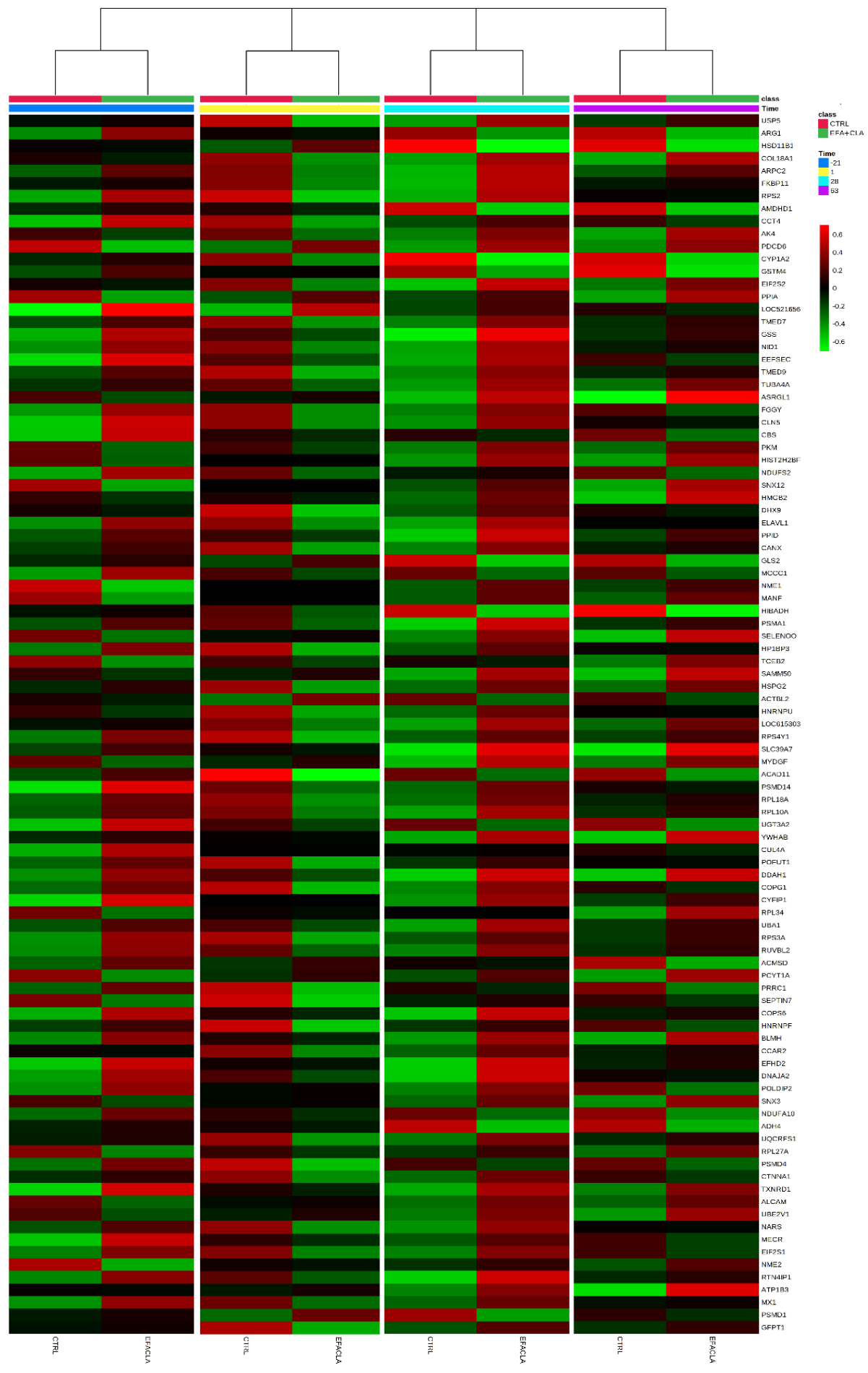

Figure 8) Hierarchical clustering and heatmap representation of differentially abundant proteins by interaction effect. Rows are the average of 
Also, the functional analyses of the DAP revealed the enrichment of nine KEGG pathways, including metabolic pathways, metabolism of xenobiotics by cytochrome P450, drug metabolism - cytochrome P450, drug metabolism other enzymes, retinol metabolism, steroid hormone biosynthesis, ribosome, chemical carcinogenesis, proteasome (Figure 9) which were mainly the same pathways found enriched for the DAP in the FA-supplemented group ( $\beta$ ). Among the KEGG enriched pathways, ribosome (with seven DAP including different ribosomal proteins (RP) S2, S3A, L34, L18A, L10A, L27A, and S4Y1), metabolism of xenobiotics by cytochrome P450 (with five DAP, CYP1A2, UGT2B4, alcohol dehydrogenase 4 (ADH4), hydroxysteroid 11-ßeta dehydrogenase 1 (HSD11B1), and GSTM4), and proteasome (with four hits including different proteasome subunits, PSMD1, PSMD4, PSMA1, and PSMD14) were the top enriched ones. In line with current results, a previous in vitro study on human liver microsomes indicated an inhibitory effect of PUFA containing linoleic acid, $\alpha$-linolenic acid, arachidonic acid, eicosapentaenoic acid and, docosahexaenoic acid on CYP1A2 [58]. In contrast, the inhibition of CYP1A2 was not observed in an in vivo study when linseed oil was infused directly into the abomasum of dairy cows [59]. However, the authors concluded that the infusion might not have achieved sufficient concentrations to inhibit the key enzymes involved in steroid metabolism. The enrichment of ribosome and proteasome pathways is probably due to elevated protein biosynthesis and turnover in the PP-cluster2 and the EFA+CLA group.

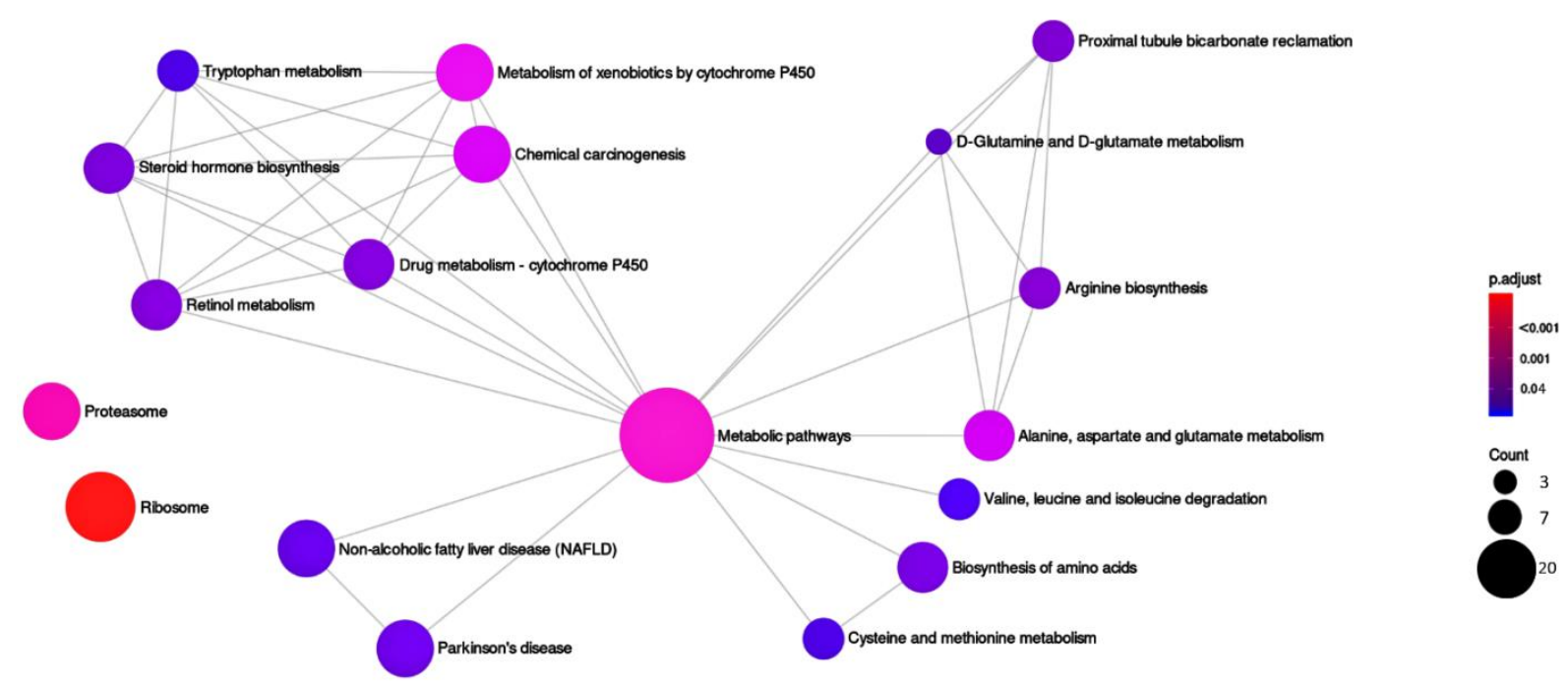

Figure 9) Kyoto Encyclopedia of Genes and Genomes (KEGG) pathway enrichment analysis of proteins identified by the interaction effect. The colour of the dots represents the $-\log 10$ (adjusted P-value); the size of the dots represents the number of differentially abundant proteins in the pathway.

Metabolism of xenobiotics by cytochrome P450 was commonly enriched during the transition to lactation ( $\alpha$ ), between treatments $(\beta)$, and interaction of them $(\alpha \beta)$, and thus can likely be considered as central mechanisms responsible for maintaining the metabolic homeostasis in response to NEFA mobilization and FA supplementation. Given that CYP are involved in the metabolism of both endogenous and exogenous substrates, it could be speculated that supplemented FA and their intermediate metabolites had xenobiotic-like potential and induced a series of reactions initiated by the 
ligand activation of PPAR. Consequently, CYP enzymes and their associated pathways such as retinol and glutathione metabolism and steroid hormone biosynthesis were being activated to regulate lipid homeostasis. However, further studies are required to verify this notion.

\subsection{Comparison of ASCA with PLS-DA method}

Choosing a suitable statistical model is always challenging, and it is related to the specific purpose of the study. ASCA design is perfectly suited for time course issues, although sometimes it would be a good complement for classical methods (i.e., PLS-DA) to provide extra information on additive effects that remained uncovered. This is because each method answers a specific question of your study. In this regard, by applying splitting in time PLS-DA, we focused explicitly on the molecular signature of the FA supplementation at each timepoint. Although, repeated measurements ASCA method entirely separated the additive effects of transition time ( $\alpha$ ), FA treatment ( $\beta$ ), and most importantly, their interaction effect $(\alpha \beta)$, which is not computable with the other methods (even considering the consecutive PLS-DA(s) on each variable separately). These separations provided us with extra information and a clear view of how FA reacted to or was affected by metabolic adaptations during the transition period.

\section{Conclusion}

The present results revealed the molecular signature of metabolic shifts during the transition from gestation to lactation in dairy cows and its interaction with supplemented EFA+CLA using the repeated measurement ASCA model. During the transition from gestation to lactation, DAP enriched metabolic pathways were mainly related to FA metabolism and degradation, AA metabolism, biosynthesis and degradation, and carbohydrate and energy metabolism in favor of energy production. Herein, the NEFA ligand activation of the nuclear PPAR orchestrates lipid metabolism, involving regulation of hepatic mitochondrial and peroxisome metabolism. Supplemented EFA+CLA amplified FA oxidation mechanisms induced by NEFA. The enrichment of cytochrome P450 as an interaction effect was to maintain metabolic homeostasis by oxidation/detoxifying endogenous and exogenous produced xenobiotics. Collectively, it could be concluded that EFA+CLA supplementation in dairy cows having a low level of these two FA, had some marginal beneficial effects on hepatic lipid metabolism and metabolic health.

\section{Acknowledgements}

The authors acknowledge A. Delavaud (INRAE) for its technical assistance in proteins extraction, quantification, and concentration for mass spectrometry analyses and R. Furioso Ferreira for drawing Figure 5.

\section{$493 \quad$ Funding}

494 This project has received funding from the European Union's Horizon 2020 research and innovation programme 495 H2020-MSCA- ITN-2017- EJD: Marie Skłodowska-Curie Innovative Training Networks (European Joint Doctorate) 
498 Data availability

499 The data and related analyses are available through the link https://doi.org/10.15454/Z2K0OR. 
Figure 1) Schematic diagram of the (A) study design, (B) proteomics workflow and peptide identification, and (C) statistical analysis and bioinformatics pipeline. (A) Timeline of supplementation (from $-63 \mathrm{~d}$ ante to $+63 \mathrm{~d}$ postpartum) and liver biopsy collection $(-21 \mathrm{~d},+1 \mathrm{~d},+28 \mathrm{~d}$, and $+63 \mathrm{~d}$ relative to parturition). Bold lines indicate liver biopsy sampling time points. (B) High-resolution LC-MS/MS analysis, peptide alignment (progenesis), and protein identification (mascot) procedure were performed by Progenesis software coupled with the Mascot search engine, statistical analysis was based on Multivariate Analysis of variance - simultaneous component analysis (ASCA), and (C) ASCA design and bioinformatics analysis.

Figure 2) Major patterns associated with transition time (A), FA treatment (B) and their interaction (C) calculated by analysis of variance simultaneous component analysis (ASCA), in dairy cows supplemented with or without EFA+CLA in 4 time-points $(-21,+1,+28$, and $+63 \mathrm{~d}$ relative to parturition. The $\mathrm{x}$-axis indicates the scores and the $\mathrm{y}$ axis indicates the variables (different timepoints (a), CTRL and EFA+CLA (b), and interaction of them $(a b)$.

Figure 3) Hierarchical clustering and heatmap representation of differentially abundant proteins during the transition from late gestation to lactation in dairy cows. Rows are respectively sorted by similarity as indicated by the left (proteins) dendrograms. Red and green represent increased and decreased protein abundance, respectively. The colour code for different time points and treatments is provided on the right-hand side.

Figure 4) Kyoto Encyclopedia of Genes and Genomes (KEGG) pathway enrichment analysis of differentially abundant proteins (DAP) during the transition from late gestation to lactation in dairy cows. The colour of the nodes represents the $-\log 10$ (adjusted P-value); Node size represents the number of DAP contained in the node (smaller indicates lesser DAP, bigger indicates more DAP).

Figure 5) Schematic of fatty acid oxidation in dairy cows' hepatocyte. In the pathway map, only the differentially abundant proteins in the postpartum period are highlighted; red colour indicates upregulation; green designated downregulation.

Figure 6) Hierarchical clustering and heatmap presentation of differentially abundant proteins between CTRL and EFA+CLA. Rows are respectively sorted by similarity as indicated by the left (proteins) dendrograms. Red and green represent increased and decreased proteins abundance, respectively. The colour code for different time points and treatments is provided on the right-hand side. CTRL and EFA+CLA. The colour of the dots represents the -log 10 (adjusted P-value); the size of the dots represents the number of DAP in the pathway.

Figure 8) Hierarchical clustering and heatmap representation of differentially abundant proteins by interaction effect. Rows are the average of protein abundaces in each group at each timepoint. Red and green represent increased and decreased protein abundance, respectively. The colour pathway. 
541 Table 1) The differentially abundant proteins identified during the time, between treatment groups, and their interaction.

\begin{tabular}{|c|c|c|c|}
\hline Num. & Protein & Gene name & $\begin{array}{c}\text { Differentially abundant } \\
\text { at }(\alpha, \beta, \alpha \beta)^{*}\end{array}$ \\
\hline 1 & Acetyl-CoA acyltransferase 1 & ACAA1 & $\alpha$ \\
\hline 2 & Acetyl-CoA acyltransferase 2 & ACAA2 & $\alpha$ \\
\hline 3 & Acyl-CoA dehydrogenase, C-4 to C-12 straight chain & ACADM & $\alpha$ \\
\hline 4 & Acetyl-CoA acetyltransferase 1 & ACAT1 & $\alpha$ \\
\hline 5 & Aconitase 2 & $\mathrm{ACO} 2$ & $\alpha$ \\
\hline 6 & Acyl-CoA thioesterase 8 & АCOT8 & $\alpha$ \\
\hline 7 & Acyl-CoA oxidase 1 & ACOX1 & $\alpha$ \\
\hline 8 & Acyl-CoA oxidase 2 & $\operatorname{ACOX} 2$ & $\alpha$ \\
\hline 9 & Acyl-CoA synthetase long-chain family member 1 & ACSL1 & $\alpha$ \\
\hline 10 & Acyl-CoA synthetase long-chain family member 5 & ACSL5 & $\alpha$ \\
\hline 11 & Acyl-CoA synthetase short-chain family member 3 & ACSS3 & $\alpha$ \\
\hline 12 & Aldo-keto reductase family 1 member D1 & AKR1D1 & $\alpha$ \\
\hline 13 & Aldehyde dehydrogenase 3 family member A2 & ALDH3A2 & $\alpha$ \\
\hline 14 & Alpha-methylacyl-CoA racemase & AMACR & $\alpha$ \\
\hline 15 & Annexin A4 & ANXA4 & $\alpha$ \\
\hline 16 & Annexin A9 & ANXA9 & $\alpha$ \\
\hline 17 & Argininosuccinate synthase 1 & ASS1 & $\alpha$ \\
\hline 18 & AU RNA binding methylglutaconyl-CoA hydratase & AUH & $\alpha$ \\
\hline 19 & Branched chain keto acid dehydrogenase $\mathrm{E} 1$, alpha polypeptide & BCKDHA & $\alpha$ \\
\hline 20 & Retinyl ester hydrolase type 1 & BREH1 & $\alpha$ \\
\hline 21 & Basigin & BSG & $\alpha$ \\
\hline 22 & Complement $\mathrm{C} 1 \mathrm{q}$ binding protein & $\mathrm{C} 1 \mathrm{QBP}$ & $\alpha$ \\
\hline 23 & Carbonyl reductase 4 & CBR4 & $\alpha$ \\
\hline 24 & Conglutinin & CGN1 & $\alpha$ \\
\hline 25 & Coenzyme Q6, monooxygenase & COQ6 & $\alpha$ \\
\hline 26 & Carnitine palmitoyltransferase 2 & CPT2 & $\alpha$ \\
\hline 27 & Carnitine O-acetyltransferase & CRAT & $\alpha$ \\
\hline 28 & Cysteine sulfinic acid decarboxylase & CSAD & $\alpha$ \\
\hline 29 & Cytochrome P450, family 27 , subfamily A, polypeptide 1 & CYP27A1 & $\alpha$ \\
\hline 30 & Cytochrome P450, family 2, subfamily E, polypeptide 1 & CYP2E1 & $\alpha$ \\
\hline 31 & Cytochrome P450, family 51, subfamily A, polypeptide 1 & CYP51A1 & $\alpha$ \\
\hline 32 & Dehydrogenase/reductase 1 & DHRS1 & $\alpha$ \\
\hline 33 & Dehydrogenase/reductase & DHRS4 & $\alpha$ \\
\hline 34 & Dihydrolipoamide S-succinyltransferase & DLST & $\alpha$ \\
\hline 35 & Dimethylglycine dehydrogenase & DMGDH & $\alpha$ \\
\hline 36 & Enoyl-CoA hydratase, short chain 1 & ECHS1 & $\alpha$ \\
\hline 37 & Enoyl-CoA hydratase and 3-hydroxyacyl CoA dehydrogenase & EHHADH & $\alpha$ \\
\hline 38 & Esterase D & ESD & $\alpha$ \\
\hline 39 & Electron transfer flavoprotein dehydrogenase & ETFDH & $\alpha$ \\
\hline 40 & Farnesyl-diphosphate farnesyltransferase 1 & FDFT1 & $\alpha$ \\
\hline
\end{tabular}




\begin{tabular}{|c|c|c|}
\hline 41 & Farnesyl diphosphate synthase & FDPS \\
\hline 42 & Ferritin light chain & FTL \\
\hline 43 & Glutamate dehydrogenase 1 & GLUD1 \\
\hline 44 & Glycerate kinase & GLYCTK \\
\hline 45 & Glutamic-oxaloacetic transaminase 1 & GOT1 \\
\hline 46 & Glycerol-3-phosphate dehydrogenase 1 & GPD1 \\
\hline 47 & Glucose-6-phosphate isomerase & GPI \\
\hline 48 & Glutathione S-transferase mu 3 & GSTM3 \\
\hline 49 & 2-hydroxyacyl-CoA lyase 1 & HACL1 \\
\hline 50 & Hydroxyacid oxidase & HAO1 \\
\hline 51 & Hydroxyacid oxidase 2 & $\mathrm{HAO} 2$ \\
\hline & 3-hydroxy-3-methylglutaryl-CoA synthase 1 & HMGCS1 \\
\hline & Hemopexin & HPX \\
\hline & Hydroxysteroid 17-beta dehydrogenase 4 & HSD17B4 \\
\hline 5 & Hydroxysteroid 17-beta dehydrogenase 8 & HSD17B8 \\
\hline 56 & $\begin{array}{l}\text { Hydroxy-delta-5-steroid dehydrogenase, } 3 \text { beta- and steroid delta- } \\
\text { isomerase } 7\end{array}$ & HSD3B7 \\
\hline 57 & Hydroxysteroid dehydrogenase like 2 & HSDL2 \\
\hline 58 & Heat shock protein family D & HSPD1 \\
\hline 59 & Isocitrate dehydrogenase & IDH1 \\
\hline 60 & Isocitrate dehydrogenase & $\mathrm{IDH} 2$ \\
\hline 61 & Isopentenyl-diphosphate delta isomerase 1 & IDI1 \\
\hline 62 & Immunoglobulin heavy constant mu & IGHM \\
\hline 63 & L-2-hydroxyglutarate dehydrogenase & L2HGDH \\
\hline 64 & $\operatorname{lamin} \mathrm{A} / \mathrm{C}$ & LMNA \\
\hline 65 & Phylloquinone omega-hydroxylase CYP4F2 & LOC100295883 \\
\hline 66 & Nicotinamide N-methyltransferase & LOC511161 \\
\hline 67 & Cytochrome P450 2C31 & LOC785540 \\
\hline 68 & Lon peptidase 1 , mitochondrial & LONP1 \\
\hline 69 & Lanosterol synthase & LSS \\
\hline 70 & mannosidase alpha class 2B member 1 & MAN2B1 \\
\hline 71 & Malate dehydrogenase 2 & MDH2 \\
\hline 72 & Malectin & MLEC \\
\hline 73 & Malonyl-CoA decarboxylase & MLYCD \\
\hline 74 & Methylsterol monooxygenase 1 & MSMO1 \\
\hline 75 & Mevalonate diphosphate decarboxylase & MVD \\
\hline 76 & Nicotinamide phosphoribosyltransferase & NAMPT \\
\hline 77 & NAD & NSDHL \\
\hline 78 & Propionyl-CoA carboxylase alpha subunit & PCCA \\
\hline 79 & Propionyl-CoA carboxylase beta subunit & PCCB \\
\hline 80 & Phosphoenolpyruvate carboxykinase 1 & PCK1 \\
\hline 81 & Peroxisomal trans-2-enoyl-CoA reductase & PECR \\
\hline 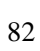 & Peroxisomal biogenesis factor 14 & PEX14 \\
\hline 8. & Phosphoglycerate dehydrogenase & PHGDH \\
\hline & Phytanoyl-CoA 2-hydroxylase & PHYH \\
\hline
\end{tabular}


RRBP1 $\alpha$

Sterol carrier protein 2

Selenium binding protein 1

SCP2

SELENBP1 $\alpha$

Sideroflexin 1

Serine hydroxymethyltransferase 2

SFXN1

SHMT2

Solute carrier family 22

SLC22A9

Solute carrier family 25 member 1

SLC25A1

SLC25A13

Solute carrier family 25 member 13

SLC25A4

Solute carrier family 25 member 4

SQLE

Squalene epoxidase

Signal transducer and activator of transcription 3

STAT3

Sulfotransferase family, cytosolic, 1A, phenol-preferring, member 1

SULT1A1

SULT1E1

THOP1

THYN1

TRAP1

TXNL1

UPP2

UQCRC1

UQCRC2

ATAD3

DDAH1

HSD11B1

$\alpha, \alpha \beta$

LOC615303

$\alpha, \alpha \beta$

MECR

$\alpha, \alpha \beta$

GSTM4

$\alpha, \alpha \beta$

FAHD2A

$\alpha, \beta$

SLC27A2

$\alpha, \beta$

UGT1A1

$\alpha, \beta$

ACAD11

$\alpha \beta$

Acyl-CoA dehydrogenase family member 11

ACMSD

$\alpha \beta$

ACTBL2

$\alpha \beta$

Actin, beta like 2

ADH4

$\alpha \beta$

Adenylate kinase 4

AK4

$\alpha \beta$

Activated leukocyte cell adhesion molecule

ALCAM

$\alpha \beta$

Amidohydrolase domain containing 1

AMDHD1

$\alpha \beta$

Arginase 1

ARG1

$\alpha \beta$

Actin related protein $2 / 3$ complex subunit 2

ARPC2

$\alpha \beta$

Asparaginase like 1

ATPase $\mathrm{Na}+\mathrm{K}+$ transporting subunit beta 3

ASRGL1

$\alpha \beta$

ATP1B3

$\alpha \beta$

BLMH

$\alpha \beta$ 


\begin{tabular}{|c|c|c|c|}
\hline 129 & Calnexin & CANX & $\alpha \beta$ \\
\hline 130 & Cystathionine-beta-synthase & CBS & $\alpha \beta$ \\
\hline 131 & Cell cycle and apoptosis regulator 2 & CCAR2 & $\alpha \beta$ \\
\hline 132 & Chaperonin containing TCP1 subunit 4 & CCT4 & $\alpha \beta$ \\
\hline 133 & Ceroid-lipofuscinosis, neuronal 5 & CLN5 & $\alpha \beta$ \\
\hline 134 & Collagen type XVIII alpha 1 chain & COL18A1 & $\alpha \beta$ \\
\hline 135 & Coatomer protein complex subunit gamma 1 & COPG1 & $\alpha \beta$ \\
\hline 136 & COP9 signalosome subunit 6 & COPS6 & $\alpha \beta$ \\
\hline 137 & Catenin alpha 1 & CTNNA1 & $\alpha \beta$ \\
\hline 138 & Cullin 4A & CUL4A & $\alpha \beta$ \\
\hline 139 & Cytoplasmic FMR1 interacting protein 1 & CYFIP1 & $\alpha \beta$ \\
\hline 140 & Cytochrome P450, family 1, subfamily A, polypeptide 2 & CYP1A2 & $\alpha \beta$ \\
\hline 141 & DExH-box helicase 9 & DHX9 & $\alpha \beta$ \\
\hline 142 & DnaJ heat shock protein family & DNAJA2 & $\alpha \beta$ \\
\hline 143 & Eukaryotic elongation factor, selenocysteine-tRNA specific & EEFSEC & $\alpha \beta$ \\
\hline 144 & EF-hand domain family member D2 & EFHD2 & $\alpha \beta$ \\
\hline 145 & Eukaryotic translation initiation factor 2 subunit alpha & EIF2S1 & $\alpha \beta$ \\
\hline 146 & Eukaryotic translation initiation factor 2 subunit beta & EIF2S2 & $\alpha \beta$ \\
\hline 147 & ELAV like RNA binding protein 1 & ELAVL1 & $\alpha \beta$ \\
\hline 148 & FGGY carbohydrate kinase domain containing & FGGY & $\alpha \beta$ \\
\hline 149 & FK506 binding protein 11 & FKBP11 & $\alpha \beta$ \\
\hline 150 & Glutamine--fructose-6-phosphate transaminase 1 & GFPT1 & $\alpha \beta$ \\
\hline 151 & Glutaminase 2 & GLS2 & $\alpha \beta$ \\
\hline 152 & Glutathione synthetase & GSS & $\alpha \beta$ \\
\hline 153 & 3-hydroxyisobutyrate dehydrogenase & HIBADH & $\alpha \beta$ \\
\hline 154 & Histone cluster $2, \mathrm{H} 2 \mathrm{bf}$ & HIST2H2BF & $\alpha \beta$ \\
\hline 155 & High mobility group box 2 & HMGB2 & $\alpha \beta$ \\
\hline 156 & Heterogeneous nuclear ribonucleoprotein F & HNRNPF & $\alpha \beta$ \\
\hline 157 & Heterogeneous nuclear ribonucleoprotein $\mathrm{U}$ & HNRNPU & $\alpha \beta$ \\
\hline 158 & Heterochromatin protein 1 binding protein 3 & HP1BP3 & $\alpha \beta$ \\
\hline 159 & Heparan sulfate proteoglycan 2 & HSPG2 & $\alpha \beta$ \\
\hline 160 & Cytochrome P450, family 2 , subfamily $\mathrm{J}$ & LOC521656 & $\alpha \beta$ \\
\hline 161 & Mesencephalic astrocyte derived neurotrophic factor & MANF & $\alpha \beta$ \\
\hline 162 & Methylcrotonoyl-CoA carboxylase 1 & MCCC1 & $\alpha \beta$ \\
\hline 163 & MX dynamin like GTPase 1 & MX1 & $\alpha \beta$ \\
\hline 164 & Myeloid derived growth factor & MYDGF & $\alpha \beta$ \\
\hline 165 & Asparaginyl-tRNA synthetase & NARS & $\alpha \beta$ \\
\hline 166 & NADH:ubiquinone oxidoreductase subunit A10 & NDUFA10 & $\alpha \beta$ \\
\hline 167 & NADH:ubiquinone oxidoreductase core subunit S2 & NDUFS2 & $\alpha \beta$ \\
\hline 168 & Nidogen 1 & NID1 & $\alpha \beta$ \\
\hline 169 & Non-metastatic cells 2 , protein & NME2 & $\alpha \beta$ \\
\hline 170 & Phosphate cytidylyltransferase 1 , choline, alpha & PCYT1A & $\alpha \beta$ \\
\hline 171 & Programmed cell death 6 & PDCD6 & $\alpha \beta$ \\
\hline 172 & Pyruvate kinase, muscle & PKM & $\alpha \beta$ \\
\hline
\end{tabular}




\begin{tabular}{|c|c|c|c|}
\hline 173 & Protein O-fucosyltransferase 1 & POFUT1 & $\alpha \beta$ \\
\hline 174 & DNA polymerase delta interacting protein 2 & POLDIP2 & $\alpha \beta$ \\
\hline 175 & Peptidylprolyl isomerase A & PPIA & $\alpha \beta$ \\
\hline 176 & Peptidylprolyl isomerase D & PPID & $\alpha \beta$ \\
\hline 177 & Proline rich coiled-coil 1 & PRRC1 & $\alpha \beta$ \\
\hline 178 & Proteasome subunit alpha 1 & PSMA1 & $\alpha \beta$ \\
\hline 179 & Proteasome $26 \mathrm{~S}$ subunit, non-ATPase 1 & PSMD1 & $\alpha \beta$ \\
\hline 180 & Proteasome 26S subunit, non-ATPase 14 & PSMD14 & $\alpha \beta$ \\
\hline 181 & Proteasome $26 \mathrm{~S}$ subunit, non-ATPase 4 & PSMD4 & $\alpha \beta$ \\
\hline 182 & Ribosomal protein L10a & RPL10A & $\alpha \beta$ \\
\hline 183 & Ribosomal protein L18a & RPL18A & $\alpha \beta$ \\
\hline 184 & Ribosomal protein L27a & RPL27A & $\alpha \beta$ \\
\hline 185 & Ribosomal protein L34 & RPL34 & $\alpha \beta$ \\
\hline 186 & Ribosomal protein S2 & RPS2 & $\alpha \beta$ \\
\hline 187 & Ribosomal protein S3A & RPS3A & $\alpha \beta$ \\
\hline 188 & Ribosomal protein S4, Y-linked 1 & RPS4Y1 & $\alpha \beta$ \\
\hline 189 & Reticulon 4 interacting protein 1 & RTN4IP1 & $\alpha \beta$ \\
\hline 190 & RuvB like AAA ATPase 2 & RUVBL2 & $\alpha \beta$ \\
\hline 191 & SAMM50 sorting and assembly machinery component & SAMM50 & $\alpha \beta$ \\
\hline 192 & Selenoprotein $\mathrm{O}$ & SELENOO & $\alpha \beta$ \\
\hline 193 & Solute carrier family 39 member 7 & SLC39A7 & $\alpha \beta$ \\
\hline 194 & Sorting nexin 12 & SNX12 & $\alpha \beta$ \\
\hline 195 & Sorting nexin 3 & SNX3 & $\alpha \beta$ \\
\hline 196 & Transcription elongation factor B subunit 2 & TCEB2 & $\alpha \beta$ \\
\hline 197 & Transmembrane p24 trafficking protein 7 & TMED7 & $\alpha \beta$ \\
\hline 198 & Transmembrane emp24 protein transport domain containing 9 & TMED9 & $\alpha \beta$ \\
\hline 199 & Tubulin alpha $4 \mathrm{a}$ & TUBA4A & $\alpha \beta$ \\
\hline 200 & Thioredoxin reductase 1 & TXNRD1 & $\alpha \beta$ \\
\hline 201 & Ubiquitin like modifier activating enzyme 1 & UBA1 & $\alpha \beta$ \\
\hline 202 & Ubiquitin conjugating enzyme E2 V1 & UBE2V1 & $\alpha \beta$ \\
\hline 203 & UDP glycosyltransferase family 3 member A2 & UGT3A2 & $\alpha \beta$ \\
\hline 204 & Ubiquinol-cytochrome c reductase, Rieske iron-sulfur polypeptide 1 & UQCRFS1 & $\alpha \beta$ \\
\hline 205 & Ubiquitin specific peptidase 5 & USP5 & $\alpha \beta$ \\
\hline 206 & $\begin{array}{l}\text { Tyrosine 3-monooxygenase/tryptophan 5-monooxygenase activation } \\
\text { protein beta }\end{array}$ & YWHAB & $\alpha \beta$ \\
\hline 207 & NME/NM23 Nucleoside Diphosphate Kinase 1 & NME1-1 & $\alpha \beta$ \\
\hline 208 & Septin 7 & SEPTIN7 & $\alpha \beta$ \\
\hline 209 & Aldo-keto reductase family 1 member A1 & AKR1A1 & $\beta$ \\
\hline 210 & Aldo-keto reductase family 7 member A2 & AKR7A2 & $\beta$ \\
\hline 211 & Aspartate beta-hydroxylase & ASPH & $\beta$ \\
\hline 212 & Caspase 6 & CASP6 & $\beta$ \\
\hline 213 & Citrate lyase beta like & CLYBL & $\beta$ \\
\hline 214 & Cystathionine gamma-lyase & $\mathrm{CTH}$ & $\beta$ \\
\hline 215 & Cathepsin A & CTSA & $\beta$ \\
\hline 216 & Cathepsin B & CTSB & $\beta$ \\
\hline
\end{tabular}




\begin{tabular}{|c|c|c|c|}
\hline 217 & Cathepsin C & CTSC & $\beta$ \\
\hline 218 & Cytochrome $\mathrm{P} 450$, family 4 , subfamily F, polypeptide 2 & CYP4F2 & $\beta$ \\
\hline 219 & L-xylulose reductase-like & DCXR & $\beta$ \\
\hline 220 & Elastin microfibril interfacer 1 & EMILIN1 & $\beta$ \\
\hline 221 & G elongation factor mitochondrial 1 & GFM1 & $\beta$ \\
\hline 222 & Gamma-glutamylamine cyclotransferase & GGACT & $\beta$ \\
\hline 223 & Growth hormone inducible transmembrane protein & GHITM & $\beta$ \\
\hline 224 & Gap junction protein beta 1 & GJB1 & $\beta$ \\
\hline 225 & Glutathione-disulfide reductase & GSR & $\beta$ \\
\hline 226 & Vigilin & HDLBP & $\beta$ \\
\hline 227 & Heterogeneous nuclear ribonucleoprotein D & HNRNPD & $\beta$ \\
\hline 228 & Aflatoxin B1 aldehyde reductase member 3 & LOC788425 & $\beta$ \\
\hline 229 & Methyltransferase like 7B & METTL7B & $\beta$ \\
\hline 230 & Metadherin & MTDH & $\beta$ \\
\hline 231 & $\begin{array}{l}\text { Methylenetetrahydrofolate dehydrogenase, cyclohydrolase and } \\
\text { formyltetrahydrofolate synthetase } 1\end{array}$ & MTHFD1 & $\beta$ \\
\hline 232 & NAD(P)H-hydrate epimerase & NAXE & $\beta$ \\
\hline 233 & N-ribosyldihydronicotinamide:quinone reductase & NQO2 & $\beta$ \\
\hline 234 & Pyruvate dehydrogenase & PDHB & $\beta$ \\
\hline 235 & Proteasome activator subunit 1 & PSME1 & $\beta$ \\
\hline 236 & RAS like proto-oncogene A & RALA & $\beta$ \\
\hline 237 & Retinol saturase & RETSAT & $\beta$ \\
\hline 238 & Selenocysteine lyase & SCLY & $\beta$ \\
\hline 239 & Serine carboxypeptidase 1 & SCPEP1 & $\beta$ \\
\hline 240 & SEC14 like lipid binding 4 & SEC14L4 & $\beta$ \\
\hline 241 & Sirtuin 5 & SIRT5 & $\beta$ \\
\hline 242 & Syntaxin binding protein 2 & STXBP2 & $\beta$ \\
\hline 243 & Sulfotransferase family, cytosolic, 2A, dehydroepiandrosterone & SULT2A1 & $\beta$ \\
\hline 244 & Thioesterase superfamily member 4 & THEM4 & $\beta$ \\
\hline 245 & Triosephosphate isomerase 1 & TPI1 & $\beta$ \\
\hline 246 & Alpha tocopherol transfer protein & TTPA & $\beta$ \\
\hline 247 & Glutathione S-Transferase Alpha 1 & GSTA1 & $\beta$ \\
\hline 248 & Cysteinyl-TRNA Synthetase 1 & CARS1 & $\beta$ \\
\hline
\end{tabular}

\section{References}

[1] J.P. Goff, R.L. Horst, Physiological changes at parturition and their relationship to metabolic disorders, J Dairy Sci 80(7) (1997) 1260-8.

[2] C. Weber, C. Hametner, A. Tuchscherer, B. Losand, E. Kanitz, W. Otten, S.P. Singh, R.M. Bruckmaier, F. 
[4] L. Rui, Energy metabolism in the liver, Compr Physiol 4(1) (2014) 177-197.

[5] M. Bionaz, S. Chen, M.J. Khan, J.J. Loor, Functional Role of PPARs in Ruminants: Potential Targets for FineTuning Metabolism during Growth and Lactation, PPAR Res. 2013 (2013) 684159.

[6] T.L. Chandler, R.T. Fugate, J.A. Jendza, A. Troescher, H.M. White, Conjugated linoleic acid supplementation during the transition period increased milk production in primiparous and multiparous dairy cows, Anim. Feed Sci. Technol. 224 (2017) 90-103.

[7] M. Zachut, A. Arieli, H. Lehrer, L. Livshitz, S. Yakoby, U. Moallem, Effects of increased supplementation of n3 fatty acids to transition dairy cows on performance and fatty acid profile in plasma, adipose tissue, and milk fat, $\mathrm{J}$ Dairy Sci 93(12) (2010) 5877-5889.

[8] D. Brockman, X. Chen, Proteomics in the characterization of adipose dysfunction in obesity, Adipocyte 1(1) (2012) 25-37.

[9] L.D. Fonseca, J.P. Eler, M.A. Pereira, A.F. Rosa, P.A. Alexandre, C.T. Moncau, F. Salvato, L. Rosa-Fernandes, G. Palmisano, J.B.S. Ferraz, H. Fukumasu, Liver proteomics unravel the metabolic pathways related to Feed Efficiency in beef cattle, Sci Rep. 9(1) (2019) 5364.

[10] M.H. Ghaffari, K. Schuh, J. Kules, N. Guillemin, A. Horvatic, V. Mrljak, P.D. Eckersall, G. Dusel, C. Koch, H. Sadri, H. Sauerwein, Plasma proteomic profiling and pathway analysis of normal and overconditioned dairy cows during the transition from late pregnancy to early lactation, J Dairy Sci 103(5) (2020) 4806-4821.

[11] B. Kuhla, K.L. Ingvartsen, Proteomics and the Characterization of Fatty Liver Metabolism in Early Lactation Dairy Cows, in: A.M. de Almeida, D. Eckersall, I. Miller (Eds.), Proteomics in Domestic Animals: from Farm to Systems Biology, Springer International Publishing, Cham, 2018, pp. 219-231.

[12] A.K. Smilde, J.J. Jansen, H.C. Hoefsloot, R.J. Lamers, J. van der Greef, M.E. Timmerman, ANOVAsimultaneous component analysis (ASCA): a new tool for analyzing designed metabolomics data, Bioinformatics 21(13) (2005) 3043-8.

[13] M. Ringner, What is principal component analysis?, Nat Biotechnol 26(3) (2008) 303-4.

[14] M.D. Wood, L.E.R. Simmatis, J. Gordon Boyd, S.H. Scott, J.A. Jacobson, Using principal component analysis to reduce complex datasets produced by robotic technology in healthy participants, J Neuroeng Rehabil 15(1) (2018) 71.

[15] Y. Xu, S.J. Fowler, A. Bayat, R. Goodacre, Chemometrics models for overcoming high between subject variability: applications in clinical metabolic profiling studies, Metabolomics 10(3) (2014) 375-385.

[16] C. Bertinetto, J. Engel, J. Jansen, ANOVA simultaneous component analysis: A tutorial review, Analytica Chimica Acta: X 6 (2020) 100061.

[17] M.E. Timmerman, H.C.J. Hoefsloot, A.K. Smilde, E. Ceulemans, Scaling in ANOVA-simultaneous component analysis, Metabolomics 11(5) (2015) 1265-1276.

[18] L. Vogel, M. Gnott, C. Kröger-Koch, D. Dannenberger, A. Tuchscherer, A. Tröscher, H. Kienberger, M. Rychlik, A. Starke, L. Bachmann, H.M. Hammon, Effects of abomasal infusion of essential fatty acids together with conjugated linoleic acid in late and early lactation on performance, milk and body composition, and plasma metabolites in dairy cows, J Dairy Sci 103(8) (2020) 7431-7450.

[19] A. Veshkini, Gene ontology of hepatic differentially abundant proteins during the transition to lactation, between different fatty acid treatments, and their interaction in Holstein cows, Portail Data INRAE, 2021. [20] J.J. Jansen, H.C.J. Hoefsloot, J. van der Greef, M.E. Timmerman, J.A. Westerhuis, A.K. Smilde, ASCA: analysis of multivariate data obtained from an experimental design, J. Chemom. 19(9) (2005) 469-481.

[21] L. Xu, L. Shi, L. Liu, R. Liang, Q. Li, J. Li, B. Han, D. Sun, Analysis of Liver Proteome and Identification of Critical Proteins Affecting Milk Fat, Protein, and Lactose Metabolism in Dariy Cattle with iTRAQ, Proteomics 19(12) (2019) e1800387.

[22] R. Liang, B. Han, Q. Li, Y. Yuan, J. Li, D. Sun, Using RNA sequencing to identify putative competing endogenous RNAs (ceRNAs) potentially regulating fat metabolism in bovine liver, Sci Rep 7(1) (2017) 6396. [23] F.B. Almughlliq, Y.Q. Koh, H.N. Peiris, K. Vaswani, O. Holland, S. Meier, J.R. Roche, C.R. Burke, M.A. Crookenden, B.J. Arachchige, S. Reed, M.D. Mitchell, Circulating exosomes may identify biomarkers for cows at risk for metabolic dysfunction, Sci Rep 9(1) (2019) 13879.

[24] K.M. Moyes, E. Bendixen, M.C. Codrea, K.L. Ingvartsen, Identification of hepatic biomarkers for physiological imbalance of dairy cows in early and mid lactation using proteomic technology, J Dairy Sci 96(6) (2013) 3599-610.

[25] P. Rawson, C. Stockum, L. Peng, B. Manivannan, K. Lehnert, H.E. Ward, S.D. Berry, S.R. Davis, R.G. Snell, D. McLauchlan, T.W. Jordan, Metabolic proteomics of the liver and mammary gland during lactation, J Proteome 75(14) (2012) 4429-35. 
[26] E. Tomaszewska, S. Świątkiewicz, A. Arczewska-Włosek, D. Wojtysiak, P. Dobrowolski, P. Domaradzki, I. Świetlicka, J. Donaldson, M. Hułas-Stasiak, S. Muszyński, Alpha-Ketoglutarate: An Effective Feed Supplement in Improving Bone Metabolism and Muscle Quality of Laying Hens: A Preliminary Study, Animals (Basel) 10(12) (2020).

[27] W. Dai, Q. Wang, F. Zhao, J. Liu, H. Liu, Understanding the regulatory mechanisms of milk production using integrative transcriptomic and proteomic analyses: improving inefficient utilization of crop by-products as forage in dairy industry, BMC Genomics 19(1) (2018) 403.

[28] J.J. Loor, R.E. Everts, M. Bionaz, H.M. Dann, D.E. Morin, R. Oliveira, S.L. Rodriguez-Zas, J.K. Drackley, H.A. Lewin, Nutrition-induced ketosis alters metabolic and signaling gene networks in liver of periparturient dairy cows, Physiol Genomics 32(1) (2007) 105-16.

[29] D. Xu, Z. Wang, Y. Xia, F. Shao, W. Xia, Y. Wei, X. Li, X. Qian, J.H. Lee, L. Du, Y. Zheng, G. Lv, J.S. Leu, H. Wang, D. Xing, T. Liang, M.C. Hung, Z. Lu, The gluconeogenic enzyme PCK1 phosphorylates INSIG1/2 for lipogenesis, Nature 580(7804) (2020) 530-535.

[30] Q. Zhang, S.L. Koser, B.J. Bequette, S.S. Donkin, Effect of propionate on mRNA expression of key genes for gluconeogenesis in liver of dairy cattle, J Dairy Sci 98(12) (2015) 8698-709.

[31] C. Agca, R.B. Greenfield, J.R. Hartwell, S.S. Donkin, Cloning and characterization of bovine cytosolic and mitochondrial PEPCK during transition to lactation, Physiol Genomics 11(2) (2002) 53-63.

[32] R.B. Greenfield, M.J. Cecava, S.S. Donkin, Changes in mRNA expression for gluconeogenic enzymes in liver of dairy cattle during the transition to lactation, J Dairy Sci 83(6) (2000) 1228-36.

[33] H.M. White, The Role of TCA Cycle Anaplerosis in Ketosis and Fatty Liver in Periparturient Dairy Cows, Animals (Basel) 5(3) (2015) 793-802.

[34] M. Wilbanks, K. Gust, S. Atwa, I. Sunesara, D. Johnson, C.Y. Ang, S. Meyer, E. Perkins, Validation of a Genomics-Based Hypothetical Adverse Outcome Pathway: 2,4-Dinitrotoluene Perturbs PPAR Signaling Thus Impairing Energy Metabolism and Exercise Endurance, Toxicolo Sci. 141 (2014).

[35] K. Banasik, J.M. Justesen, M. Hornbak, N.T. Krarup, A.P. Gjesing, C.H. Sandholt, T.S. Jensen, N. Grarup, A. Andersson, T. Jorgensen, D.R. Witte, A. Sandbaek, T. Lauritzen, B. Thorens, S. Brunak, T.I. Sorensen, O. Pedersen, T. Hansen, Bioinformatics-driven identification and examination of candidate genes for non-alcoholic fatty liver disease, PLoS One 6(1) (2011) e16542.

[36] L. Shi, L. Liu, Z. Ma, X. Lv, C. Li, L. Xu, B. Han, Y. Li, F. Zhao, Y. Yang, D. Sun, Identification of genetic associations of ECHS1 gene with milk fatty acid traits in dairy cattle, Anim Genet 50(5) (2019) 430-438.

[37] S. Busato, M. Bionaz, The interplay between non-esterified fatty acids and bovine peroxisome proliferatoractivated receptors: results of an in vitro hybrid approach, J. Anim. Sci 11(1) (2020) 91.

[38] L.M. Sanderson, T. Degenhardt, A. Koppen, E. Kalkhoven, B. Desvergne, M. Muller, S. Kersten, Peroxisome proliferator-activated receptor beta/delta (PPARbeta/delta) but not PPARalpha serves as a plasma free fatty acid sensor in liver, Mol Cell Biol 29(23) (2009) 6257-67.

[39] M. Fransen, C. Lismont, P. Walton, The Peroxisome-Mitochondria Connection: How and Why?, Int J Mol Sci 18(6) (2017).

[40] F. Schroeder, B.P. Atshaves, A.L. McIntosh, A.M. Gallegos, S.M. Storey, R.D. Parr, J.R. Jefferson, J.M. Ball, A.B. Kier, Sterol carrier protein-2: new roles in regulating lipid rafts and signaling, Biochim Biophys Acta 1771(6) (2007) 700-18.

[41] C.K. Reynolds, P.C. Aikman, B. Lupoli, D.J. Humphries, D.E. Beever, Splanchnic Metabolism of Dairy Cows During the Transition From Late Gestation Through Early Lactation, J. Dairy Sci. 86(4) (2003) 1201-1217.

[42] M. Larsen, N.B. Kristensen, Precursors for liver gluconeogenesis in periparturient dairy cows, animal 7(10) (2013) 1640-1650.

[43] A.E. Harper, R.H. Miller, K.P. Block, Branched-chain amino acid metabolism, Annu Rev Nutr 4 (1984) 40954.

[44] T.R. Mackle, D.A. Dwyer, D.E. Bauman, Effects of branched-chain amino acids and sodium caseinate on milk protein concentration and yield from dairy cows, J Dairy Sci 82(1) (1999) 161-71.

[45] H. Sadri, D. von Soosten, U. Meyer, J. Kluess, S. Danicke, B. Saremi, H. Sauerwein, Plasma amino acids and metabolic profiling of dairy cows in response to a bolus duodenal infusion of leucine, PLoS One 12(4) (2017) $\mathrm{e} 0176647$.

[46] A.G. Wessels, H. Kluge, F. Hirche, A. Kiowski, A. Schutkowski, E. Corrent, J. Bartelt, B. Konig, G.I. Stangl, High Leucine Diets Stimulate Cerebral Branched-Chain Amino Acid Degradation and Modify Serotonin and Ketone Body Concentrations in a Pig Model, PLoS One 11(3) (2016) e0150376.

[47] P. Anzenbacher, E. Anzenbacherova, Cytochromes P450 and metabolism of xenobiotics, Cell Mol Life Sci 58(5-6) (2001) 737-47. 
[48] U.M. Zanger, M. Schwab, Cytochrome P450 enzymes in drug metabolism: regulation of gene expression, enzyme activities, and impact of genetic variation, Pharmacol Ther 138(1) (2013) 103-41.

[49] R.D. Finn, C.J. Henderson, C.L. Scott, C.R. Wolf, Unsaturated fatty acid regulation of cytochrome P450 expression via a CAR-dependent pathway, Biochem J 417(1) (2009) 43-54.

[50] J. Kato, A. Ikemoto, T. Mizutani, The Effect of Dietary Fatty Acids on the Expression Levels and Activities of Hepatic Drug Metabolizing Enzymes, J. Health Sci. 49(2) (2003) 105-114.

[51] C. Shi, L. Min, J. Yang, M. Dai, D. Song, H. Hua, G. Xu, F.J. Gonzalez, A. Liu, Peroxisome ProliferatorActivated Receptor alpha Activation Suppresses Cytochrome P450 Induction Potential in Mice Treated with Gemfibrozil, Basic Clin Pharmacol Toxicol 121(3) (2017) 169-174.

[52] L.L. Grasfeder, S. Gaillard, S.R. Hammes, O. Ilkayeva, C.B. Newgard, R.B. Hochberg, M.A. Dwyer, C.Y. Chang, D.P. McDonnell, Fasting-induced hepatic production of DHEA is regulated by PGC-1alpha, ERRalpha, and HNF4alpha, Mol Endocrinol 23(8) (2009) 1171-82.

[53] M.S. Salleh, G. Mazzoni, J.K. Hoglund, D.W. Olijhoek, P. Lund, P. Lovendahl, H.N. Kadarmideen, RNA-Seq transcriptomics and pathway analyses reveal potential regulatory genes and molecular mechanisms in high- and lowresidual feed intake in Nordic dairy cattle, BMC Genomics 18(1) (2017) 258.

[54] C.O. Lemley, S.T. Butler, W.R. Butler, M.E. Wilson, Short communication: insulin alters hepatic progesterone catabolic enzymes cytochrome P450 2C and 3A in dairy cows, J Dairy Sci 91(2) (2008) 641-5.

[55] L. Vogel, M. Gnott, C. Kröger-Koch, S. Gors, J.M. Weitzel, E. Kanitz, A. Hoeflich, A. Tuchscherer, A. Tröscher, J.J. Gross, R.M. Bruckmaier, A. Starke, L. Bachmann, H.M. Hammon, Glucose metabolism and the somatotropic axis in dairy cows after abomasal infusion of essential fatty acids together with conjugated linoleic acid during late gestation and early lactation, J Dairy Sci 104(3) (2021) 3646-3664.

[56] J.D. Hayes, J.U. Flanagan, I.R. Jowsey, Glutathione transferases, Annu. Rev. Pharmacol. Toxicol. 45 (2005) 51-88.

[57] A. Abuelo, J. Hernández, J.L. Benedito, C. Castillo, Oxidative stress index (OSi) as a new tool to assess redox status in dairy cattle during the transition period, Animal 7(8) (2013) 1374-8.

[58] H.T. Yao, Y.W. Chang, S.J. Lan, C.T. Chen, J.T. Hsu, T.K. Yeh, The inhibitory effect of polyunsaturated fatty acids on human CYP enzymes, Life Sci 79(26) (2006) 2432-40.

[59] C.A. Piccinato, R. Sartori, S. Sangsritavong, A.H. Souza, R.R. Grummer, D. Luchini, M.C. Wiltbank, In vitro and in vivo analysis of fatty acid effects on metabolism of 17beta-estradiol and progesterone in dairy cows, J Dairy Sci 93(5) (2010) 1934-43.

[60] A. Veshkini, H. M. Hammon, L. Vogel, M. Delosi`ere, D. Viala, S. D`ejean, A. Troscher, ” F. Ceciliani, H. Sauerwein, M. Bonnet, Liver proteome profiling in dairy cows during the transition from gestation to lactation: effects of supplementation with essential fatty acids and conjugated linoleic acids as explored by PLS-DA, J. Proteomics (2022), https://doi.org/10.1016/j. jprot.2021.104436. In press. 